\title{
Strong Light-Matter Interaction in Lithography-Free Planar Metamaterial Perfect Absorbers
}

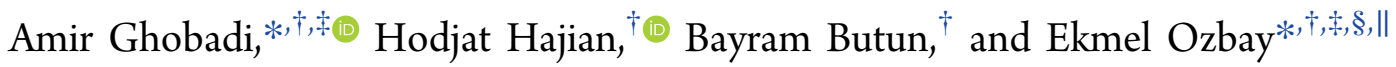 \\ ${ }^{\dagger}$ NANOTAM-Nanotechnology Research Center, Bilkent University, 06800 Ankara, Turkey \\ ${ }^{\ddagger}$ Department of Electrical and Electronics Engineering, Bilkent University, 06800 Ankara, Turkey \\ ${ }^{\S}$ Department of Physics, Bilkent University, 06800 Ankara, Turkey \\ "UNAM-Institute of Materials Science and Nanotechnology, Bilkent University, Ankara, Turkey
}

ABSTRACT: The efficient harvesting of electromagnetic (EM) waves by subwavelength nanostructures can result in perfect light absorption in the narrow or broad frequency range. These metamaterial-based perfect light absorbers are of particular interest in many applications, including thermal photovoltaics, photovoltaics, sensing, filtering, and photodetection applications. Although advances in nanofabrication have provided the opportunity to observe strong light-matter interaction in various optical nanostructures, the repeatability and upscaling of these nano units have remained a challenge for their use in large scale applications. Thus, in recent years,

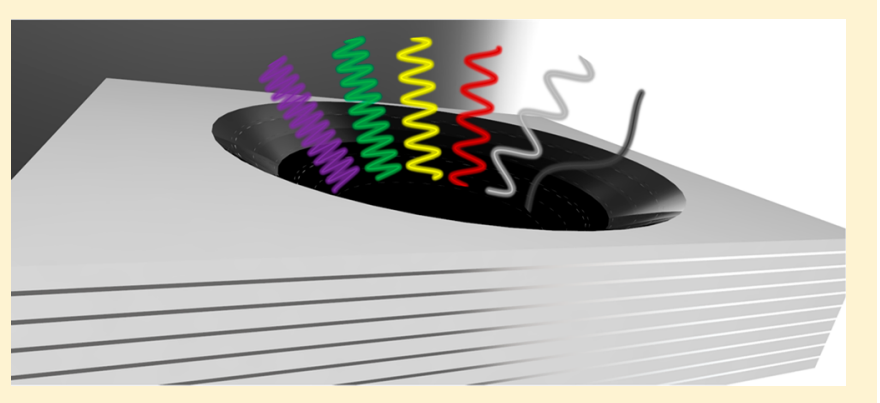
the concept of lithography-free planar light perfect absorbers has attracted much attention in different parts of the EM spectrum, owing to their ease of fabrication and high functionality. This Perspective explores the material and architecture requirements for the realization of light perfect absorption using these multilayer metamaterial designs from ultraviolet (UV) to far-infrared (FIR) wavelength regimes. We provide a general theoretical formulation to find the ideal condition for achieving near unity light absorption. Later, these theoretical estimations are coupled with findings of recent studies on perfect light absorbers to explore the physical phenomena and the limits of different materials and design architectures. These studies are categorized in three main class of materials; metals, semiconductors, and other types of materials. We show that, by the use of proper material and design configuration, it is possible to realize these lithography-free light perfect absorbers in every portion of the EM spectrum. This, in turn, opens up the opportunity of the practical application of these perfect absorbers in large scale dimensions. In the last section, we discuss the progress, challenges, and outlook of this field to outline its future direction.

KEYWORDS: metamaterials, perfect absorber, metal-insulator multilayer, broadband absorption, plasmonic

M etamaterials refer to a class of synthetic materials comprising designed inclusions that offer exotic properties. Negative refraction, ${ }^{1-3}$ artificial magnetism, ${ }^{4,5}$ asymmetric transmission, ${ }^{6,7}$ lasing, ${ }^{8,9}$ cloak of invisibility, ${ }^{10-12}$ and subwavelength light absorption ${ }^{13}$ are examples of these attributes. The concept of light confinement and harvesting by subwavelength geometries has been one of the most intensively studied areas in recent years. Efficient harvesting of this confined field utilizing an absorbing layer such as metal, or semiconductor material could lead to the realization of perfect absorption of light. These light perfect absorbers can be employed in a vast variety of applications. Metal-based perfect absorbers can afford their response in narrow or broad spectral ranges. The narrowband metal perfect absorbers are of particular interest in sensing, imaging, and color filter applications. ${ }^{14-21}$ While their broadband counterparts have potential applications in thermal photovoltaics, ${ }^{22-24}$ radiative cooling, ${ }^{25-27}$ hot electron-based photodetectors, ${ }^{28,29}$ photochemistry, $^{30}$ and efficient solar vapor/steam generation. ${ }^{31-33}$ Similar to metals, ultrathin light perfect absorbers can be also realized using semiconductor based configurations where a strong light-matter interaction can harvest most of the incident light photons and go beyond the Yablonovitch limit in an ultrathin thickness. ${ }^{34}$ The generation of high density photo carriers in dimensions smaller than the semiconductor diffusion length can guarantee their efficient collection. Therefore, these semiconductor based metamaterials are promising choices for future performance enhanced ultrathin optoelectronics. In recent years, resonant light trapping schemes were employed in different applications including ultrathin photovoltaic solar cells, spectrally selective photodetectors, and other optoelectronic applications. ${ }^{28,35-45}$ These perfect absorbers have also been realized in other types of materials, such as polar and two-dimensional (2D) materials, that is, graphene, transition metal dichalcogenides (TMDs), and black phosphorus, that will be discussed in the following.

Received: June 28, 2018

Revised: October 10, 2018

Accepted: October 21, 2018

Published: October 30, 2018 
Although there are enormous efforts being made in the performance enhancement of these nanostructured perfect absorbers, the fabrication complexity of these nanostructures hinders their further upscaling.

In recent years, extensive attention has been paid to the design of lithography-free multilayer perfect absorbers of light. Strong light-matter interaction in these properly designed planar structures can provide perfect absorption in narrow or broad wavelength ranges while keeping the overall process large scale compatible. The goal of this Perspective is to provide a comprehensive overview of these planar multilayer designs in three different classes of materials; metals, semiconductors, and other type of materials. It is shown that by choosing proper material and design architecture, it is possible to obtain strong light absorption in different ranges of electromagnetic (EM) spectrum. The lithography-free nature of these designed multilayer structures provides great flexibility and high-throughput manufacturing convenience and opens a new opportunity for low-cost, high performance, and robust optoelectronic devices. It should be mentioned that there are some omissions for the topics and designs discussed here. For instance, novel synthesis methods such as chemical or selfassembly techniques can be utilized to obtain lithography-free perfect absorbers. However, this Perspective mainly focuses on subwavelength planar designs that can be fabricated with common cleanroom equipment with high fabrication throughput and repeatability. Finally, in the last section, we discuss the progress, challenges, and the outlook of this field to outline its future direction.

\section{THEORETICAL BACKGROUND}

In order to realize nearly perfect absorption of the light, reflectance should be suppressed by matching the effective impedance of the metamaterial to that of the incident medium. Simultaneously, transmittance is eliminated by introducing another metallic plate acting as a mirror for metamaterial absorbers. In this section, an alternative analytical-numerical approach based on transfer matrix method (TMM) will be provided to predict the most appropriate permittivity of the lossy layers, labeled $\mathrm{X}$, in a metamaterial absorber that comprises $\mathrm{N}$ two-layer elementary IX unit cells, that is, $M(I X)^{N}$. The studied $M(I X)^{N}$ design configuration is schematically shown in Scheme 1. In this definition, M, I, and $\mathrm{X}$ stand for metal mirror, insulator, and lossy medium. The lossy layer could be metal, semiconductor, or any other types of absorbing materials. We can investigate the ideal permittivity $\left(\varepsilon_{\mathrm{X}}\right)$ of the lossy layers $(\mathrm{X})$ in the $\mathrm{M}(\mathrm{IX})^{\mathrm{N}}$ structure through an analytical-numerical approach based on TMM. Supposing that the M(IX) $)^{\mathrm{N}}$ metamaterial is bounded with air $\left(\varepsilon_{\mathrm{a}}\right)$ and a substrate $\left(\varepsilon_{\mathrm{s}}\right)$, and considering $z=0$ plane as the interface of air the first insulator layer, the $y$ component of the magnetic field can be written as
Scheme 1. Schematic Representation of the Studied M(IX) $)^{\mathrm{N}}$ Design to Find the Ideal Condition for Light Perfect Absorption

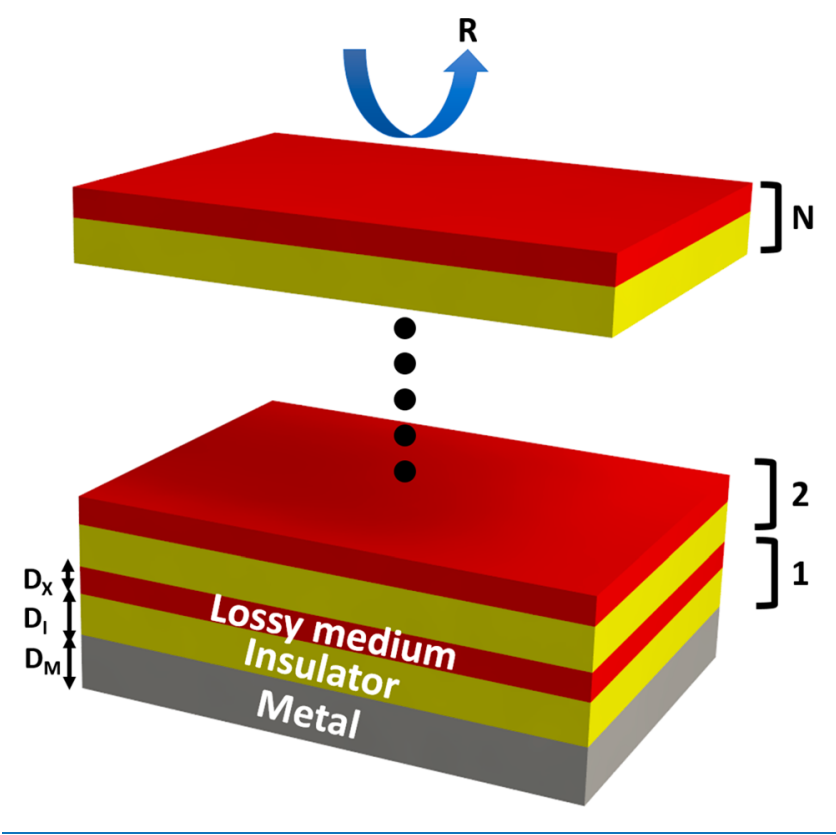

$$
H_{y}(z)=\left\{\begin{array}{c}
A_{i} e^{i k_{\mathrm{a}} z}+A_{r} e^{-i k_{\mathrm{a}} z}, \quad z<0 \\
I_{11} e^{i k_{\mathrm{I}} z}+I_{12} e^{-i k_{\mathrm{I}} Z}, \quad 0<z<D_{\mathrm{I}} \\
\mathrm{X}_{11} e^{i k_{\mathrm{X}}\left(z-D_{\mathrm{I}}\right)}+\mathrm{X}_{12} e^{-i k_{\mathrm{X}}\left(z-D_{\mathrm{I}}\right)}, \quad D_{\mathrm{I}}<z<\left(D_{\mathrm{I}}+D_{\mathrm{X}}\right) \\
I_{21} e^{i k_{\mathrm{I}}\left[z-\left(D_{\mathrm{I}}+D_{\mathrm{X}}\right)\right]}+I_{22} e^{-i k_{\mathrm{I}}\left[z-\left(D_{\mathrm{I}}+D_{\mathrm{X}}\right)\right]}, \quad\left(D_{\mathrm{I}}+D_{\mathrm{X}}\right)<z<L \\
: \\
\cdot \\
M_{11} e^{i k_{\mathrm{M}}[z-N L]}+M_{12} e^{-i k_{\mathrm{M}}[z-N L]}, \quad N L<<\left(N L+D_{\mathrm{M}}\right) \\
S_{t} e^{i k_{\mathrm{S}}\left[z-\left(N L+D_{\mathrm{M}}\right)\right]}, \quad z>\left(N L+D_{\mathrm{M}}\right)
\end{array}\right\}
$$

where $L=2 D_{\mathrm{I}}+D_{\mathrm{X}}$ and $k_{j=(\mathrm{a}, \mathrm{X}, \mathrm{I}, \mathrm{M}, \mathrm{s})}=\sqrt{\varepsilon_{j} \omega^{2} / c^{2}-k_{x}^{2}}$. In these formulations, $D_{\mathrm{I}}, D_{\mathrm{X}}$, and $D_{\mathrm{M}}$ are the thicknesses of the dielectric, lossy medium, and bottom metal reflector layers, respectively, $c$ is the speed of light in vacuum, $k$ is the wave vector inside different media, and $\varepsilon$ is the permittivity data of different layers. Applying the boundary conditions for transverse magnetic (TM) polarization (the continuity of the fields and their derivatives at the boundaries separating different media), reflection of the incident light from the structure can be obtained as $R=\left|F_{12} / F_{11}\right|^{2} .{ }^{46}$ Here, $F=\left[\begin{array}{l}F_{11} \\ F_{12}\end{array}\right]=a^{-1}\left(X_{1} X_{2}^{-1} I_{1} I_{2}^{-1}\right)^{N} M_{1} M_{2}^{-1} s$, where

$$
\begin{aligned}
& a=\left[\begin{array}{cc}
1 & 1 \\
i k_{\mathrm{a}} / \varepsilon_{\mathrm{a}} & -i k_{\mathrm{a}} / \varepsilon_{\mathrm{a}}
\end{array}\right], s=\left[\begin{array}{c}
1 \\
\frac{i k_{\mathrm{s}}}{\varepsilon_{\mathrm{s}}}
\end{array}\right] \\
& I_{1}=\left[\begin{array}{cc}
1 & 1 \\
i k_{\mathrm{I}} / \varepsilon_{\mathrm{I}} & -i k_{\mathrm{I}} / \varepsilon_{\mathrm{I}}
\end{array}\right], I_{2}=\left[\begin{array}{cc}
e^{i k_{\mathrm{I}} D_{\mathrm{I}}} & e^{-i k_{\mathrm{I}} D_{\mathrm{I}}} \\
i k_{\mathrm{I}} e^{i k_{\mathrm{I}} D_{\mathrm{I}}} / \varepsilon_{\mathrm{I}} & -i k_{\mathrm{I}} e^{-i k_{\mathrm{I}} D_{\mathrm{I}}} / \varepsilon_{\mathrm{I}}
\end{array}\right]
\end{aligned}
$$




$$
\begin{aligned}
X_{1} & =\left[\begin{array}{cc}
1 & 1 \\
i k_{\mathrm{X}} / \varepsilon_{\mathrm{X}} & -i k_{\mathrm{X}} / \varepsilon_{\mathrm{X}}
\end{array}\right], X_{2} \\
& =\left[\begin{array}{cc}
e^{i k_{\mathrm{X}} D_{\mathrm{X}}} & e^{-i k_{\mathrm{X}} D_{\mathrm{X}}} \\
i k_{\mathrm{X}} e^{i k_{\mathrm{X}} D_{\mathrm{x}}} / \varepsilon_{\mathrm{X}} & -i k_{\mathrm{X}} e^{-i k_{\mathrm{X}} D_{\mathrm{X}}} / \varepsilon_{\mathrm{X}}
\end{array}\right] \\
M_{1} & =\left[\begin{array}{cc}
1 & 1 \\
i k_{\mathrm{M}} / \varepsilon_{\mathrm{M}} & -i k_{\mathrm{M}} / \varepsilon_{\mathrm{M}}
\end{array}\right], M_{2} \\
& =\left[\begin{array}{cc}
e^{i k_{\mathrm{M}} D_{\mathrm{M}}} & e^{-i k_{\mathrm{M}} D_{\mathrm{M}}} \\
i k_{\mathrm{M}} e^{i k_{\mathrm{M}} D_{\mathrm{M}}} / \varepsilon_{\mathrm{M}} & -i k_{\mathrm{M}} e^{-i k_{\mathrm{M}} D_{\mathrm{M}}} / \varepsilon_{\mathrm{M}}
\end{array}\right]
\end{aligned}
$$

Considering the normal light incidence and the planar nature of the design, the same response is observed for transverse electric (TE) polarization. Knowing the thickness and epsilon of the dielectric layers and the bottom metal layer as well as the thickness of the top X layer, we can numerically obtain the real and imaginary values of $\varepsilon_{\mathrm{X}}$ under nearly perfect absorption condition (which we define as $R<0.1$ ). More explanation will be provided in the following parts of the Perspective.

Another approach to obtain nearly perfect absorption using unpatterned structures is to combine a thin film of a plasmonic/phononic lossy material with a one-dimensional photonic crystal (1D PC). In this case, Tamm plasmon/ phonon modes can be supported by the lossy-based ID PC for both TM and TE polarizations that, in turn, show themselves as nearly perfect resonant peaks (deeps) in the absorption (reflection) spectrum. Therefore, by appropriately designing the structure and obtaining the Tamm modes in the wavelength range of interest, nearly perfect absorption peaks that are supported by the system can be estimated. A typical 1D PC comprises alternating dielectric layers with permittivities $\varepsilon_{1}$ and $\varepsilon_{2}$ and thicknesses $d_{1}$ and $d_{2}$ that is separated from the lossy material by a spacer layer $\left(\varepsilon_{\mathrm{s}}\right.$ and $\left.\mathrm{d}_{\mathrm{s}}\right)$. We suppose the nonmagnetic plasmonic/phononic lossy material has thickness $t$ and, in a general form, has a uniaxially anisotropic permittivity of $\varepsilon_{\text {lossy }}=\operatorname{diag}\left(\varepsilon_{t}, \varepsilon_{t}, \varepsilon_{z}\right)$. Taking the $y$ component of a magnetic field as

$$
H_{y}(z)=\left\{\begin{array}{c}
a e^{-q_{\mathrm{a}}(z-t / 2)}, z>t / 2 \\
h_{1} e^{-q_{\mathrm{h}, \mathrm{TM}^{z}}}+h_{2} e^{q_{\mathrm{h}, \mathrm{TM}}},-t / 2 \leq z \leq t / 2 \\
s_{1} e^{-q_{\mathrm{s}}(z+t / 2)}+s_{2} e^{q_{\mathrm{s}}(z+t / 2)},-\left(t / 2+d_{\mathrm{s}}\right) \leq z \leq-t / 2 \\
p\left\{\sinh \left(q_{1}\left[z+\left(\frac{t}{2}+d_{\mathrm{s}}\right)\right]\right)+\gamma_{\mathrm{TM}} \cosh \left(q_{1}\left[z+\left(\frac{t}{2}+d_{\mathrm{s}}\right)\right]\right)\right\}, \\
z<-\left(t / 2+d_{\mathrm{s}}\right)
\end{array}\right.
$$

and applying the appropriate boundary conditions for TM polarization at different interfaces, dispersion of the Tamm modes of the 1D lossy-based PC can be obtained using the direct-matching procedure that is applicable for electronic and photonic layered structures ${ }^{47-49}$

$$
\tanh \left(q_{\mathrm{h}, \mathrm{TM}} t\right)=-\frac{\Gamma_{\mathrm{a}}+\Gamma_{\mathrm{s}}}{1+\Gamma_{\mathrm{a}} \Gamma_{\mathrm{s}}}
$$

Here, $\Gamma_{\mathrm{a}}=q_{\mathrm{h}} \varepsilon_{\mathrm{a}} / q_{\mathrm{a}} \varepsilon_{\mathrm{t}}, \quad \Gamma_{\mathrm{s}}=q_{\mathrm{h}} X_{3, \mathrm{TM}} / \varepsilon_{\mathrm{t}}, X_{3, \mathrm{TM}}=\varepsilon_{\mathrm{s}}(1+$ $\left.e^{-2 q_{\mathrm{s}} d_{\mathrm{s}}} X_{1, \mathrm{TM}}\right) / q_{\mathrm{s}} X_{2, \mathrm{TM}}, X_{2, \mathrm{TM}}=\left(-1+e^{-2 q_{\mathrm{s}} d_{\mathrm{s}}} X_{1, \mathrm{TM}}\right), X_{1, \mathrm{TM}}=\Gamma_{1}$

$$
\begin{aligned}
& +1 / \Gamma_{1}-1, \Gamma_{1}=q_{\mathrm{s}} \varepsilon_{1} \gamma_{\mathrm{TM}} / q_{1} \varepsilon_{s}, q_{j=(\mathrm{a}, \mathrm{s}, 1,2)}=\sqrt{k_{x}^{2}-\varepsilon_{j} \omega^{2} / c^{2}} \\
& q_{\mathrm{h}, \mathrm{TM}}=\varepsilon_{t} \sqrt{k_{x}^{2}-\varepsilon_{z} \omega^{2} / c^{2}} / \varepsilon_{z}, \text { and } \\
& \gamma_{\mathrm{TM}}=\frac{F_{\mathrm{TM}} e^{i K_{\mathrm{B}} d} \sinh \left(q_{2} d_{2}\right)+\sinh \left(q_{1} d_{1}\right)}{e^{i K_{\mathrm{B}} d} \cosh \left(q_{2} d_{2}\right)-\cosh \left(q_{1} d_{1}\right)}
\end{aligned}
$$

where $F_{\mathrm{TM}}=F_{1, \mathrm{TM}} / F_{2, \mathrm{TM}}$. Moreover, $e^{i K_{\mathrm{B}} d}$ can be calculated using the well-known dispersion relation of a $1 D$ PC

$$
\begin{aligned}
\cos \left(i K_{\mathrm{B}} d\right)= & \cosh \left(q_{1} d_{1}\right) \cosh \left(q_{2} d_{2}\right)+\frac{F_{1, \mathrm{TE}, \mathrm{TM}}}{F_{2, \mathrm{TE}, \mathrm{TM}}} \\
& +\frac{F_{2, \mathrm{TE}, \mathrm{TM}}}{F_{1, \mathrm{TE}, \mathrm{TM}}} \times \sinh \left(q_{1} d_{1}\right) \sinh \left(q_{2} d_{2}\right)
\end{aligned}
$$

with $F_{i, \mathrm{TM}}=\varepsilon_{i} / q_{i}, F_{i, \mathrm{TE}}=q_{i}$.

In order to have an estimation of the nearly perfect resonant absorption for TE polarization, we also formulate TE Tamm polaritons of the 1D lossy-based PC. To this aim, considering $E_{y}$ as eq 3 and applying TE boundary conditions, we arrive at the following dispersion relation for the TE Tamm modes ${ }^{48}$

$$
\tanh \left(q_{\mathrm{h}, \mathrm{TE}} t\right)=\frac{X_{4}-X_{5}}{X_{4}+X_{5}}
$$

Here, $X_{4}=\left(-q_{\mathrm{h}, \mathrm{TE}}-X_{3, \mathrm{TE}}\right)\left(q_{\mathrm{h}, \mathrm{TE}}-q_{\mathrm{a}}\right), X_{5}=\left(-q_{\mathrm{h}, \mathrm{TE}}+\right.$ $\left.X_{3, \mathrm{TE}}\right)\left(q_{\mathrm{h}, \mathrm{TE}}+q_{\mathrm{a}}\right), X_{3, \mathrm{TE}}=q_{\mathrm{s}}\left(-1+e^{-2 q_{\mathrm{s}} d_{\mathrm{s}}} X_{1, \mathrm{TE}}\right) / X_{2, \mathrm{TE}}, X_{2, \mathrm{TE}}=(1$ $\left.+e^{-2 q_{s} d_{s}} X_{1, \mathrm{TE}}\right), X_{1, \mathrm{TE}}=\left(q_{1} / \gamma_{\mathrm{TE}}+q_{\mathrm{s}}\right) /\left(-q_{1} / \gamma_{\mathrm{TE}}+q_{\mathrm{s}}\right)$, $q_{\mathrm{h}, \mathrm{TE}}=\sqrt{k_{x}^{2}-\varepsilon_{x} \omega^{2} / c^{2}}$ and $\gamma_{\mathrm{TE}}$ can be calculated using eq 3 by replacing $F_{\mathrm{TE}}$ with $F_{\mathrm{TM}}$. It should be noted that a similar approach can be followed for obtaining Tamm modes that are supported by a $1 \mathrm{D}$ PC based on $2 \mathrm{D}$ materials. ${ }^{49}$

Metal-Based Perfect Absorbers. The most commonly studied metal-insulator (MI) pair-based architecture that provides perfect light absorption is subwavelength metalinsulator-metal (MIM)-based cavity. ${ }^{21,50-56}$ In this MIM configuration, the top metal layer is a nanopatterned plasmonic metal, while the bottom layer is an optically thick mirror. In this design, due to strong light confinement inside the cavity in the resonance frequency, perfect absorption of light can be realized in dimensions much smaller than the incident light wavelength. Coupling of incident wave to collective oscillation of top metal electrons via the excitation of surface plasmon resonances (SPRs) leads to light harnessing in the desired frequency range. ${ }^{21,56}$ However, due to the fact that the SPRs can be supported in a relatively narrow frequency range, the absorption bandwidth (BW) of these plasmonic designs has a narrow spectral coverage. ${ }^{13,51,53,57-71}$ Many different design strategies have been employed to broaden the absorption BW of these plasmonic MIM cavities. One of the most frequently utilized ideas is to implement a multidimensional/multishape pattern in the top metal layer where each unit is designed in a way that SPR excitation can be obtained in different adjacent wavelengths and the superposition of these resonance modes provide perfect absorption of light in a broad frequency range. ${ }^{52,72-79}$ The use of tightly packed arrangements of lossy metal nano units, elongated shapes (such as ellipse) that support dual SPR modes, and structures with sharp corners are other alternatives to make plasmonic broadband perfect absorbers. $^{80-85}$ However, the main drawback with all of these design configurations is their complex fabrication route. To realize the top subwavelength nano units, electron beam lithography $(\mathrm{EBL})$ is required that is inherently an 

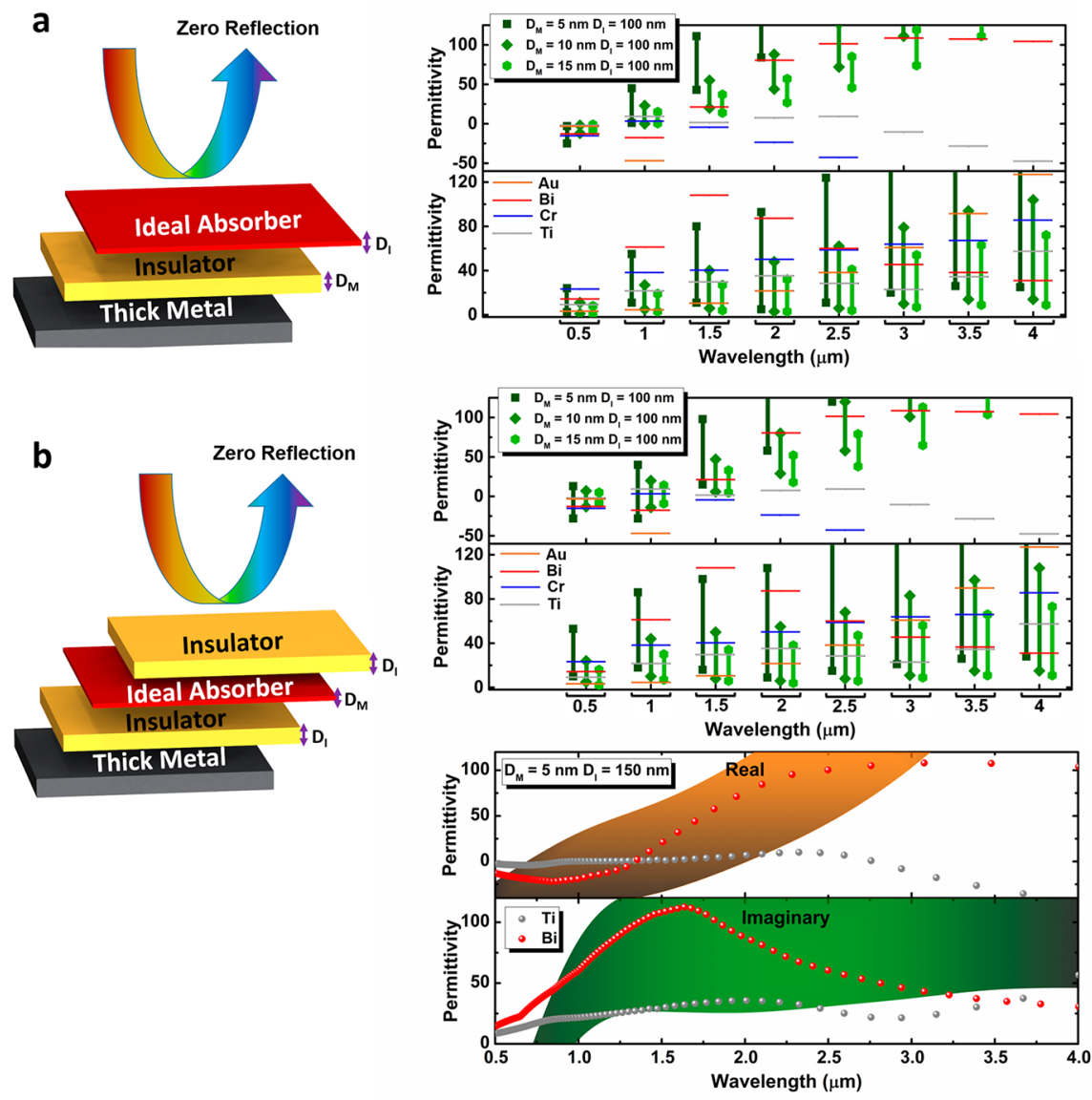

Figure 1. Ideal tolerable permittivity values for (a) MIM and (b) MIMI configurations. These ideal values (that correspond to absorption above 0.9 ) have been extracted for different $D_{\mathrm{M}}$ and $D_{\mathrm{I}}$ geometries for eight different discrete wavelength values. These tolerable ideal permittivity regions are compared with four different metals including $\mathrm{Au}, \mathrm{Bi}, \mathrm{Cr}$, and $\mathrm{Ti}$.

incompatible approach for large scale applications. Although nano imprint lithography (NIL) could be a solution for the large scale synthesis of these samples, this system is also a complex and costly one. Therefore, to open up the opportunity of practical application of these perfect absorbers, the concept of lithography-free multilayer planar designs has attracted much attention in recent years. ${ }^{86-114}$ Unlike resonant plasmonic MIM perfect absorbers, nonresonant MIM cavities that are made of lossy metals such as chromium (Cr), titanium $(\mathrm{Ti})$, tungsten $(\mathrm{W})$, and nickel $(\mathrm{Ni})$ can be used to obtain light perfect absorption in the visible (Vis) and near-infrared (NIR) wavelength regimes. ${ }^{94,115}$ In these configurations, the top planar lossy metal layer (with a thickness in the order of couple of nanometers) offers a low quality factor $\left(Q_{F}\right)$ cavity that satisfies impedance match conditions in an ultrabroadband frequency range. In this planar configuration, the use of noble metals such as gold $(\mathrm{Au})$ and silver $(\mathrm{Ag})$ could result in narrow absorption BW, which is of great interest for the realization of visible light color filters. ${ }^{87,89,90,92,111,114}$ To have a better understanding on the absorption BW limits of this configuration, above-mentioned TMM (eq 1) is adopted for an MIM cavity to find the ideal top material for nearly perfect light absorption. In this model, the bottom metal layer is fixed as an optically thick metal mirror, and the spacer layer is chosen as $\mathrm{SiO}_{2}{ }^{116}$ The tolerable real and imaginary values of permittivity data, for achieving absorption above 0.9 , are obtained for eight discrete wavelengths spanning from 0.5 to 4 $\mu \mathrm{m}$, and the results are shown in Figure 1. These ideal regimes have been extracted for three different top layer thicknesses of 5,10 , and $15 \mathrm{~nm}$, where the spacer layer thickness is fixed at $100 \mathrm{~nm}$. Being located inside the highlighted region in every wavelength value can ensure an absorption above $90 \%$ that we define as the threshold for perfect absorption of incident light. As can be seen from Figure 1a, thinner top layers have wider spans for getting perfect absorption, while thicker layers move the bottom of the ideal region toward zero. These ideal permittivity ranges are compared with those of permittivity values for different metals of $\mathrm{Au},{ }^{116}$ bismuth $(\mathrm{Bi}),{ }^{117} \mathrm{Cr},{ }^{116}$ and $\mathrm{Ti}^{116}$ As Figure 1a clearly implies, the ideal region for the real part of permittivity gets positive values as we move toward longer wavelengths. However, most of the metals (including both noble $[\mathrm{Au}]$ and lossy ones $[\mathrm{Bi}, \mathrm{Cr}, \mathrm{Ti}]$ ) take an exponential drop toward negative values in NIR wavelengths. For noble metals, such as $\mathrm{Au}$ and $\mathrm{Ag}$, this leads to a narrowband light absorber that has been widely explored in Febry-Perot color filter and sensing applications, as shown in Figure 2a,b, while for lossy metals, such as $\mathrm{Cr}$ and $\mathrm{Ti}$, this matching can be met in broader wavelength range. Generally, however, for a planar MIM absorber, the perfect light absorption is limited to visible and the beginning of the NIR regimes (roughly $\lambda<1000 \mathrm{~nm}$ ). This limit can be extended toward longer wavelength values with tuning the permittivity values of the metals, for example, composition of a metal with a low refractive index medium such as air. ${ }^{115,118,119}$ We recently adopted a method based on the dewetting process to create ultrasmall nanoholes inside a layer of $\mathrm{Cr}$ to reduce its effective 


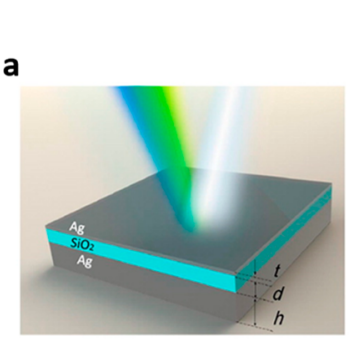

e

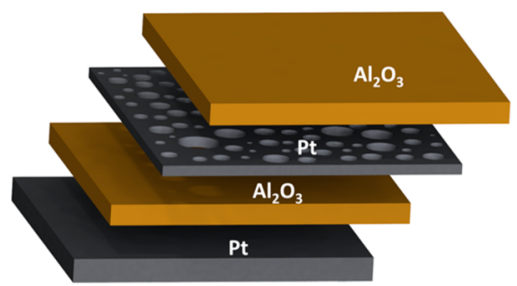

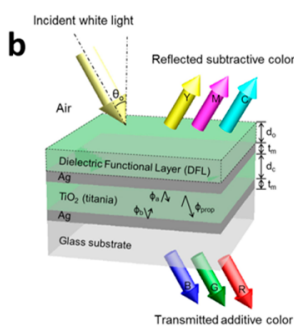

f

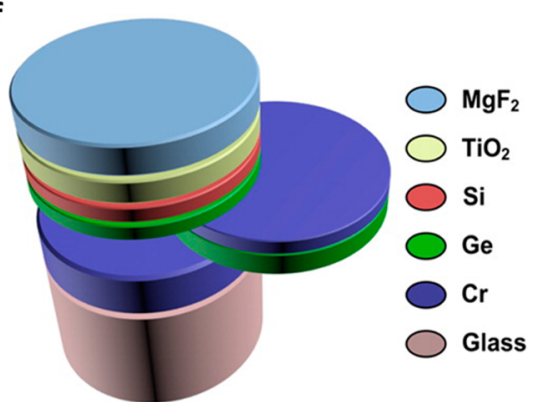

C
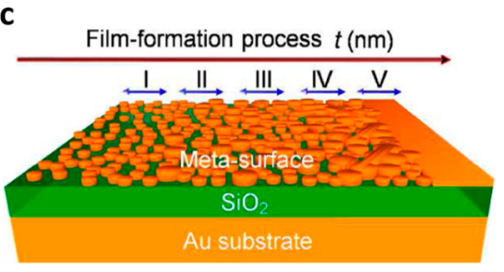

g

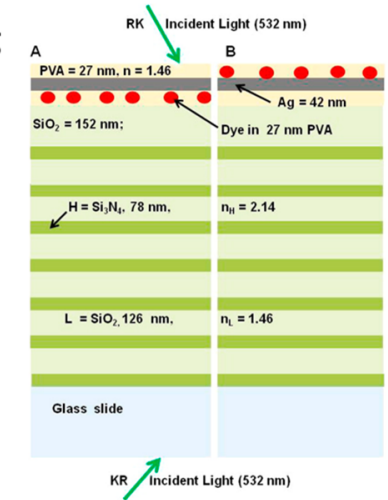

Figure 2. Narrowband light perfect absorbers by use of $\mathrm{Ag}$ in (a) $\mathrm{MIM}^{87}$ and (b) MIMI ${ }^{86}$ configurations to realize a visible light color filter. (c) Automatically acquired different shaped nano islands during the film formation process in an MIM structure to obtain broadband perfect light absorption. ${ }^{119}$ (d) Periodic arrangement of 16 pairs $\mathrm{Ni} / \mathrm{SiO}_{2}$ and $\mathrm{Ti} / \mathrm{SiO}_{2}$ (where the metal layer thickness is in the order of $1-2 \mathrm{~nm}$ ) to realize light perfect absorption up to $2.5 \mu \mathrm{m} .{ }^{102}$ (e) Formation of disordered nanohole patterns via dewetting process to realize ultrabroadband perfect light absorption in an MIMI multilayer architecture. ${ }^{106}$ (f) Properly designed multilayer perfect absorber for ultrabroadband impedance matching. ${ }^{120}(\mathrm{~g})$ Schematic for an Ag-based 1D PC with two PVA layers. The dye is either located below (case A) or above (case B) the Ag film. The support of Tamm plasmons by this structure leads to the directional emission of the emitter. ${ }^{131}$ (a) Reprinted with permission from ref 87. Copyright 2015 American Chemical Society. (b) Reprinted with permission from ref 86. Copyright 2016 Springer Nature. (c) Reprinted with permission from ref 119. Copyright 2015 American Chemical Society. (d) Reprinted with permission from ref 102 . Copyright 2016 The Optical Society. (e) Reprinted with permission from ref 106. Copyright 2017 Springer Nature. (f) Reprinted with permission from ref 120 . Copyright 2016 American Chemical Society. (g) Reprinted with permission from ref 131. Copyright 2014 American Chemical Society.

permittivity values. ${ }^{115}$ It was proven that this modification in a Cr-based MIM cavity can shift the upper absorption edge from $850 \mathrm{~nm}$ (for planar design) to $1150 \mathrm{~nm}$ (for disordered random nanohole design). Moreover, controlling metal deposition condition could be another way to create metal nanostructures, ${ }^{119}$ see Figure 2 c. Further improvement in the absorption BW of the design can be accomplished by utilizing a metal-insulator-metal-insulator (MIMI) configuration. As shown in Figure 1b, in this design, the top insulator layer acts as a broadband antireflection coating to provide a gradual impedance matching between the air and the underlying MIM cavity. Extracting the ideal data for this case, it can be seen that the overlap between the ideal perfect absorption region and the metal permittivity values have been extended toward longer wavelengths (compared to that of MIM design). ${ }^{97,99,105-107}$ In fact, this configuration can make the ideal permittivity range wider compared to that of the MIM design. However, similar to the MIM case, absorption toward longer wavelengths [midinfrared (MIR)] cannot be achieved by this configuration. Although increasing the number of pairs could substantially improve the absorption bandwidth, the upper absorption edge is still limited to the NIR regime. ${ }^{99,102-104}$ Increasing the insulator layer thickness will red-shift both the lower and the upper absorption edge in which the perfect absorption of light will not cover the visible region. ${ }^{93}$ As depicted in Figure $2 \mathrm{~d}$, it has been proven that the periodic arrangement of 16 pairs $\mathrm{Ni} /$ $\mathrm{SiO}_{2}$ and $\mathrm{Ti} / \mathrm{SiO}_{2}$ (where the metal layer thickness is in the order of $1-2 \mathrm{~nm}$ ) could provide near unity light absorption up to $2.5 \mu \mathrm{m} .{ }^{102}$ However, such a large number of pairs require multiple deposition processes and considering the ultrathin nature of metal layer, the repeatability could be an issue in this type of metamaterial absorbers. Therefore, it is desired to improve light absorption BW while keeping device dimensions intact. To achieve this goal, different strategies have been proposed. The optimum choice of back reflector, ${ }^{107}$ multithickness metal layers, ${ }^{105}$ and incorporation of randomly sized nano holes ${ }^{106}$ (Figure 2e) can further improve the absorption BW in MIMI configuration. Not only the periodic arrangement of MI pairs can provide ultrabroadband light perfect absorption but also proper arrangement of different multilayers with designed thicknesses can ensure impedance matched condition. An example of such a structure is shown in Figure 2f. ${ }^{120}$ Nevertheless, according to the ideal model, the absorption at longer wavelengths requires a positive real part of permittivity condition that cannot be satisfied with common lossy and noble metals. That is actually the reason why this configuration cannot go beyond some wavelength limits. However, Bi has extraordinary permittivity values in the longer wavelengths that are perfectly matched to the ideal region. As depicted in Figure 1b, Bi has small negative real epsilon values at wavelengths below $1.5 \mu \mathrm{m}$ and this value moves toward positive ones as we go toward longer wavelengths. Therefore, it is envisioned that by tuning the spacer and $\mathrm{Bi}$ layer's thicknesses in MIM and MIMI configurations, perfect absorption can be acquired in an ultrabroadband frequency range covering all the Vis and NIR frequencies. To prove this 
statement, the ideal tolerable region is extracted for the case of $D_{\mathrm{M}}=5 \mathrm{~nm}$, and $D_{\mathrm{I}}=150 \mathrm{~nm}$ and its matching is compared with that of permittivity data of $\mathrm{Bi}$ and $\mathrm{Ti}$ (as two metals that have positive permittivity values in long wavelengths). As it can be deduced from this figure, the perfect absorption of incident light is retained up to $3 \mu \mathrm{m}$ with MIMI dimensions in the order of $\lambda / 10$. Therefore, not only the design configuration, but also the optimum choice of the material is essential to acquire near unity absorption in an ultrabroadband frequency range. It should be mentioned that the general approach for achieving perfect light absorption in this MI pair based multilayers, throughout NIR and MIR regimes, is to use tapered structure. ${ }^{03,121-128}$ Such architecture will provide gradual matching between the air and underlying metamaterial impedance. However, these designs require lithography and etching processes that are out of the focus of this Perspective. Moreover, some other studies realized perfect light absorption by the integration of ultrathin metal with lithography-free light trapping scaffold. ${ }^{129,130}$ We recently showed that a coating of a $10 \mathrm{~nm}$ thick $\mathrm{Pt}$ layer on randomly oriented $\mathrm{TiO}_{2}$ nanowires can provide an absorption above $97 \%$ in a broad frequency range spanning from Vis to the NIR regime. ${ }^{129}$ However, in this Perspective, we essentially investigate the light absorption in planar sub wavelength structures that operate based on strong interface effects.

Another approach to obtain light perfect absorption is the integration of a thin metal layer with a 1D PC structure. Unlike an MI pair-based design configuration, this configuration could provide light absorption in a narrow frequency range and it is of particular interest in directional emission and sensing applications. In agreement with the aforementioned analytical approach for the investigation of Tamm modes supported by the lossy-based 1D PC, here, we provide some examples regarding the nearly perfect resonant absorption observed by these structures. PCs are artificial structures with dielectric constants periodically varying in one, two, or three dimensions, and display photonic band gaps, that is, the frequency regions that light cannot propagate in the structure. ${ }^{132}$ Since the pioneering work by Yablonovitch, ${ }^{133}$ control of light emission has been indicated as one of the most promising applications of PCs. 1D PC are capable of supporting surface waves that can propagate along the interface of the PC and the adjacent medium over long distances and are confined in the normal direction (these modes can also be supported in twodimensional photonic crystals (2D PCs), ${ }^{132}$ however, their investigation is not of our interest in this Perspective). Moreover, the surface waves may be considered as dielectric analogues of surface plasmons that propagate on a metal/ dielectric surface. ${ }^{134}$ Yeh et al. ${ }^{135}$ proved that a $1 \mathrm{D}$ PC is capable of supporting surface waves for both TE and TM polarizations. Due to the hybridization of those surface waves with the surface plasmons of a thin film of metal in a metalbased 1D PC, Tamm plasmons can be supported by the system. Consequently, inspired by that point, nearly perfect and directional absorption/emission was obtained by an Agbased 1D PC due to the support of Tamm plasmons (see Figure $2 \mathrm{~g}){ }^{131,136-138}$ For the structure schematically illustrated in Figure $2 \mathrm{~g}, 42 \mathrm{~nm}$ film of silver is separated from a 1D PC by $26 \mathrm{~nm}$ film of $\mathrm{SiO}_{2}$, and the 1D PC comprising 14 alternating layers of $\mathrm{SiO}_{2}(126 \mathrm{~nm})$ and $\mathrm{Si}_{3} \mathrm{~N}_{4}(78 \mathrm{~nm})$. Since the emission of poly(vinyl alcohol) material, PVA, was what was interested in, ${ }^{131,138}$ the $\mathrm{Ag}$ film is symmetrically bound with two $27 \mathrm{~nm}$ PVA layers to incorporate fluorophores. The addition of an Ag layer to the 1D PC structure leads to a dip in reflectivity in the photonic band gap (PBG) region at $637 \mathrm{~nm}$, due to excitation of Tamm plasmons. It is observed that, in this case, directional emission at normal excitation can be achieved by the structure. In return, by removing the PVA layers and impinging the light to the structure from the Ag part, directional resonant absorption can be obtained. As mentioned herein above, Tamm plasmons, that were reported for the first time in $2007,{ }^{139}$ are created due to the hybridization of surface plasmons of the thin film of $\mathrm{Ag}$ and surface waves of the 1D PC in a hybrid structure. The electric fields for the Tamm plasmons are localized in the dielectric below the metal film and can be supported for both TE and TM polarizations at normal incidences, unlike surface plasmons. Moreover, since the wavevector of Tamm plasmons is smaller than that of light in vacuum, they can be directly excited from air without the aid of prisms or gratings. Using eqs 4 and 7 and by appropriate choice of the material, geometrical parameters of the 1D PC, and the spacer layer, it is possible to obtain dispersion curves of the Tamm plamons for both TE and TM polarizations for a thin film of silver with a thickness of $t$ and $\varepsilon_{\text {lossy }}=\varepsilon_{\mathrm{Ag}}$. Since this dispersion is in exact agreement with the directional-spectral absorption/reflection of the Ag-based 1D PC, the parameters that are taken for the dispersion curves would be a fair estimation for the design and fabrication of the directional absorber. By replacing Silver with an alternative plasmonic material $^{140}$ and designing appropriate $1 \mathrm{D}$ PC, it would be possible to achieve nearly perfect resonant absorbers operating at the desired wavelength range.

Semiconductor-Based Perfect Absorbers. Metamaterials are not only promising solutions for metal-based perfect absorbers, but they can also provide strong light-matter interaction in ultrathin semiconductor-based designs. It should be noted that semiconductors can act as plasmonic (highly doped semiconductors) and phononic (lowly doped or intrinsic semiconductors) materials. A comprehensive discussion on obtaining absorption using unpatterned phononic structures will be provided in the next section. This section will focus on the strategies employed to obtain strong above optical band gap absorption in semiconductor-based metamaterial designs. A semiconductor-based metamaterial can provide strong light absorption in ultrathin layers. Therefore, near unity light absorption can be achieved in dimensions much smaller than the incident light wavelength. The absorption of light in such thin layers can also be beneficiary from the electrical perspective. Considering the short diffusion length of most of semiconductors, ${ }^{141}$ total absorption of light in nanometer scale dimensions can significantly improve the collection efficiency of these carriers by suppressing their recombination. In other words, there is a trade-off between light absorption and carrier collection for a specific semiconductor film thickness. ${ }^{142}$ The integration of metamaterial configurations to ultrathin semiconductor films is a promising approach to get high efficiency optoelectronic devices. Such a simple ultrathin trapping design configuration is able to exceed Yablonovitvh's theoretical limit. $^{34}$ Therefore, the application of these functional architectures in optoelectronics, such as photovoltaic, photo detectors, and photoelectrochemical devices has attracted a lot of attention in recent years.

Unlike metals that keep their large extinction coefficient values in a wide wavelength range, semiconductors can only absorb photons with energies above their inherent optical band gap. Therefore, they are only active at Vis and short 

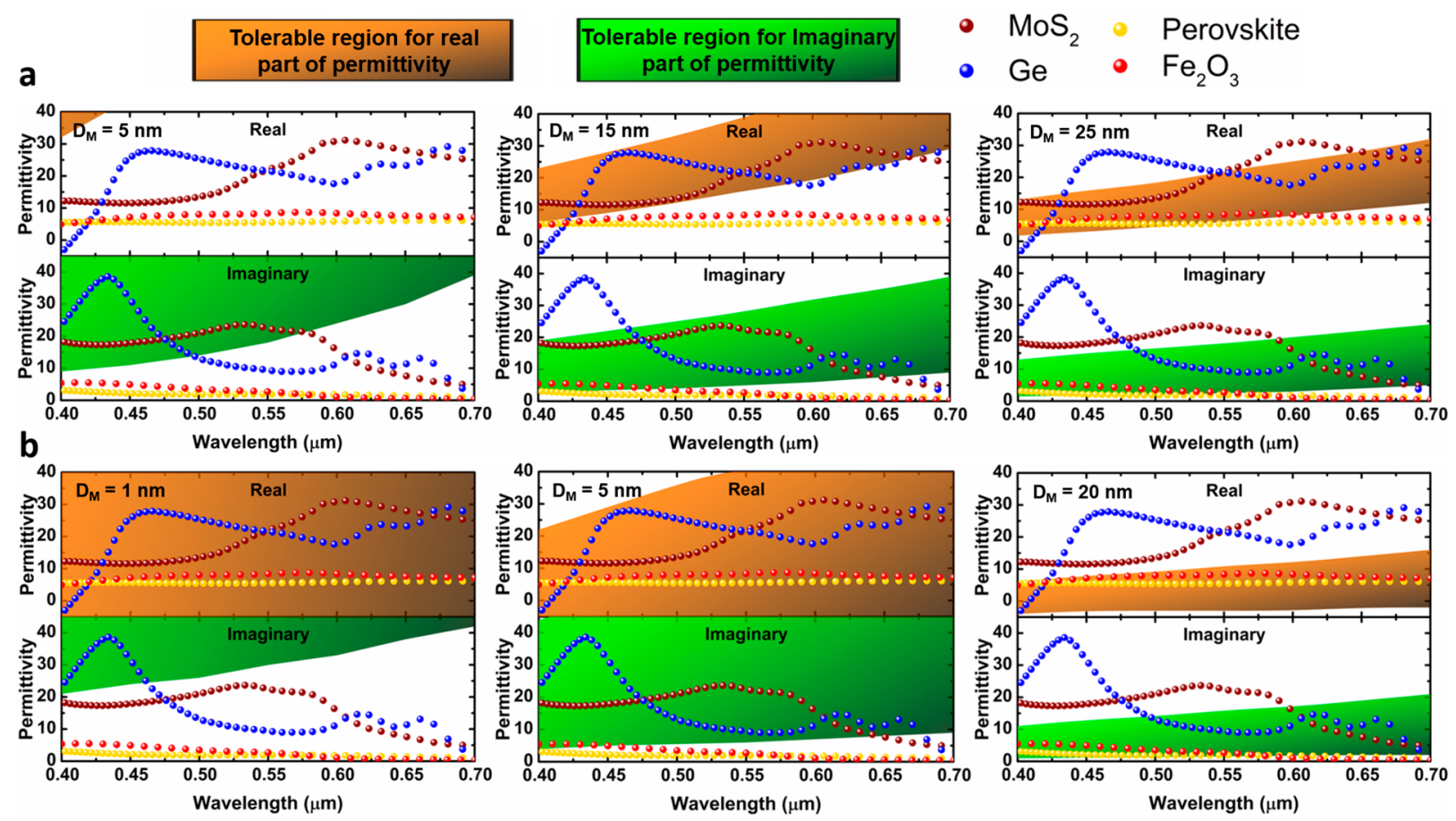

Figure 3. Matching between the permittivity values of four different semiconductors $\left(\mathrm{MoS}_{2}\right.$, Ge, perovskite, and $\left.\mathrm{Fe}_{2} \mathrm{O}_{3}\right)$ and those of ideal material to obtain strong light absorption (absorption above 0.7) for (a) MS and (b) MIS configurations. For the MIS case, the insulator layer thickness is fixed at $50 \mathrm{~nm}$.

wavelength NIR frequency ranges. The essential goal in the semiconductor based metamaterials is to increase the lightmatter interaction to enhance light harvesting efficiency. Same as the previous section, we first need to explore the condition for strong light absorption. For this aim, the ideal material permittivity values are extracted for metal-semiconductor (MS) and metal-insulator-semiconductor (MIS) configurations. Considering the low extinction coefficient of semiconductors, we chose 0.7 as the threshold for light absorption and highlighted the tolerable permittivity regions for an absorption above this value. The overlap between these ideal regions and four different materials [germanium $(\mathrm{Ge})$ as a low band gap semiconductor, monolayer of molybdenum disulfide $\left(\mathrm{MoS}_{2}\right)$ as a $2 \mathrm{D}$ semiconductor, $\mathrm{CH}_{3} \mathrm{NH}_{3} \mathrm{PbI}_{3}$ perovskite (PVSK) as an organic semiconductor, iron(III) oxide (hematite, $\mathrm{Fe}_{2} \mathrm{O}_{3}$ ) as a low band gap metal oxide] is compared in Figure 3a,b. For this goal, the ideal model is obtained for three different top material thicknesses of $D_{M}=5,15$, and 25 $\mathrm{nm}$ for MS (Figure 3a) and $D_{\mathrm{M}}=1,5$, and $20 \mathrm{~nm}$ for MIS configurations (Figure $3 \mathrm{~b}$ ), respectively. The bottom layer is chosen as an optically thick highly reflecting $\mathrm{Al}^{116}$ layer, and the spacer is chosen as a $50 \mathrm{~nm}$ thick $\mathrm{SiO}_{2}$. The simplest metamaterial design to provide strong light absorption is MS configuration. In one of the pioneer works, ${ }^{143}$ it was shown that the amorphous germanium (a-Ge) layer with a thickness below $25 \mathrm{~nm}$ coated on thick Au metal can absorb around 90\% of the light in a relatively narrow Vis frequency range. The spectral position of the absorption peak gets red shift as the thickness of the semiconducting layer increases. It was proven that this nearly perfect absorption is due to the coupling of Fabry-Perot (FP) resonances with excited Brewster modes, see Figure $4 \mathrm{a} .{ }^{34}$ Later studies have proven this strong interface effects for other ultrathin coatings, such as $\mathrm{Si}$ and $\mathrm{Fe}_{2} \mathrm{O}_{3}$. ${ }^{144-147}$ In a recent study that is schematically illustrated in Figure
$4 \mathrm{~b},{ }^{144}$ a perfect absorber made of an ultrathin Si coating on top of $\mathrm{AlCu}$ alloy was realized in the Vis-NIR frequency regime. This coating supports Brewster modes and acts as a dual band absorber where the spectral position of these peaks can be finely tuned from the Vis to the NIR by simply changing the semiconductor layer thickness. However, in the abovementioned MS structures, the perfect absorption is realized in a narrow wavelength region that is not our desire in energy related applications where higher density of absorbed photons can provide larger current values. The use of a multilayer back reflector instead of a bare metal layer in which the layers provide gradual phase matching is found to substantiate light absorption in a broad wavelength regime. ${ }^{148}$ As shown in Figure $4 c$, this strategy is successfully applied on $\mathrm{Fe}_{2} \mathrm{O}_{3}$-based water splitting photoanode in which an estimated amount of $71 \%$ of the incident photons of energy above the band gap of Hematite can be absorbed by a coating thinner than $50 \mathrm{~nm}$. Thus, most of the photogenerated holes can reach the surface and oxidize water before recombination takes place. This 1D trapping scheme can be also utilized in the design of ultrathin solar cells. ${ }^{149}$ The a-Ge:H solar cell proposed in Figure $4 \mathrm{~d}$ has an efficiency of $3.6 \%$ with an absorbing layer as thin as $13 \mathrm{~nm}$. Further improvement in the performance of these optoelectronic devices can be attained by improving the crystalline quality of the absorber layer. PVSK as a chemically synthesized ultrathin crystalline coating can be a proper option to achieve this goal. ${ }^{150-153}$ Despite all of these considerations, according to the ideal model data for a MS design at ultrathin coatings, the mismatch is generally due to the low values of imaginary permittivities of semiconductors. However, thicker layers could provide the matching for both real and imaginary parts of permittivity in a relatively narrow frequency range for all four different materials. This matching can be met in a broader frequency range for MIS configuration. In this case, similar to 

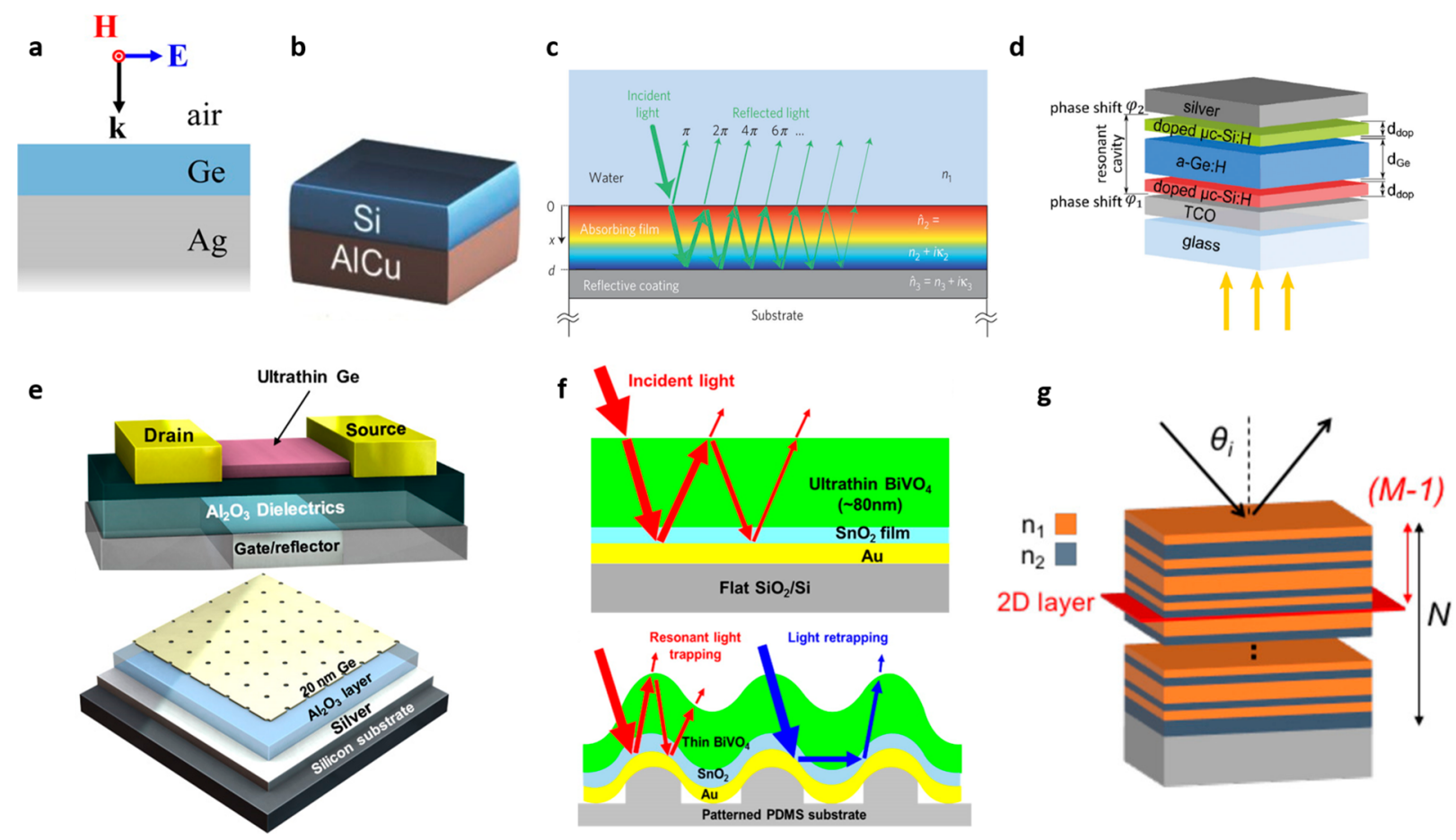

Figure 4. (a) Absorption of $98 \%$ of the incident light energy in a $12 \mathrm{~nm}$ thick Ge layer on an Ag substrate at a narrow wavelength range $(\lambda=625$ $\mathrm{nm}$ ) over a wide range of angles. ${ }^{34}$ (b) Dual band light perfect absorber operating at the Vis and NIR regimes using a $45 \mathrm{~nm}$ thick Si layer coated on $\mathrm{AlCu}$ alloy. ${ }^{144}$ (c) $71 \%$ above band gap light absorption in a less than $50 \mathrm{~nm}$ thick $\mathrm{Fe}_{2} \mathrm{O}_{3}$ using a resonant light trapping scheme for efficient water splitting. ${ }^{148}(\mathrm{~d})$ a-Ge:H solar cell with an efficiency as high as $3.6 \%$ using an absorbing layer as thin as $13 \mathrm{~nm} .{ }^{149}$ (e) Spectrally selective photodetector by the realization of perfect light absorption in a narrow frequency range using a single-crystalline germanium nanomembrane coated on a resonant foreign nanocavity. ${ }^{157}$ (f) An efficient water splitting photoanode using an ultrathin $\mathrm{BiVO}_{4}$ absorbing layer in an MIS configuration $\left(\mathrm{Au}-\mathrm{SnO}_{2}-\mathrm{BiVO}_{4}\right)$. The incorporation of a trapping scaffold could substantiate the process efficiency. ${ }^{161}(\mathrm{~g})$ Random coherent perfect absorption in a monolayer of $\mathrm{MoS}_{2}$ mediated by anderson localization. ${ }^{162}$ (a) Reprinted with permission from ref 34 . Copyright 2014 American Chemical Society. (b) Reprinted with permission from ref 144. Copyright 2018 John Wiley and Sons. (c) Reprinted with permission from ref 148 . Copyright 2012 Springer Nature. (d) Reprinted with permission from ref 149. Copyright 2015 John Wiley and Sons. (e) Reprinted with permission from ref 157. Copyright 2017 The American Association for the Advancement of Science. (f) Reprinted with permission from ref 161. Copyright 2016 American Chemical Society. (g) Reprinted with permission from ref 162. Copyright 2017 American Chemical Society.

MS structure, ultrathin layers $(\sim 1 \mathrm{~nm})$ have poor matching. Thicker layers could shift the highlighted ideal regions toward zero but this time the real part of permittivity values stays out of the tolerable ideal region. Therefore, each material has its own optimum condition for strong light absorption and by simultaneous tuning of semiconductor and spacer layers thicknesses, strong light absorption in a broad frequency range can be achieved. As for a material thickness of $1 \mathrm{~nm}$ that is around the thickness of a monolayer of a two-dimensional (2D) semiconductor, we have poor matching between the ideal case and different semiconductors. Therefore, this configuration cannot provide strong light absorption in $2 \mathrm{D}$ monolayers of semiconductors but rather it is useful in its few layer dimensions. ${ }^{154-156}$ However, for thicker top layer thickness of $5 \mathrm{~nm}$, the matching condition can be achieved in a broad frequency range for materials with relatively high extinction coefficient such as $\mathrm{Ge}$ and $\mathrm{MoS}_{2}$. Moving to larger thicknesses, this broad absorption can be acquired for weak absorbing materials such as metal oxides. Increasing the spacer layer thickness to larger values (comparable to $\lambda / 4$ ) can also tune the spectral position of the matching and, in general, a narrow absorption response will be obtained in this case. All the above-mentioned results show the potential application of this metamaterial configuration in optoelectronic applications. However, as mentioned earlier, the main problem with these lithography-free structures is the electrical behavior of the amorphous top active layer that suffers from low electron mobility and a short diffusion length. In other words, it is difficult to obtain ultrathin materials with single-crystalline quality. In a very recent work, ${ }^{157}$ authors proposed a method to fabricate single-crystalline Ge coatings in an MIS configuration and by this way spectrally selective high performance photodetectors were realized in dimensions much smaller than a bulk device, see Figure 4e. It has been proven that, by tuning the top single crystalline Ge layer thickness, the absorption peak can be tuned throughout the optical band gap of the semiconductor coating. Moreover, it has been proven that ultrathin crystalline PVSK and metal oxide layers can also be achieved using chemical synthesis methods. ${ }^{151,158,159}$ Besides the above-mentioned optoelectronic applications of these metamaterial cavities, these designs can also be promising in photoelectrochemical water splitting cells. In recent years, MIS cavity configuration, for which the insulator layer thickness is below $\sim 5 \mathrm{~nm}$ (to facilitate carrier tunneling) and the semiconductor layer is an optically thick coating, has attracted much attention. ${ }^{160}$ In this structure, light is absorbed by the semiconductor layer and the applied voltage facilitates the tunneling of the carrier toward the metal layer as well as at the surface of the metal layer, in which the hydrogen evolution reaction takes place. Considering the fact that the carrier tunneling rate exponentially drops by increasing the insulator thickness and existence of any surface trap states can 
a
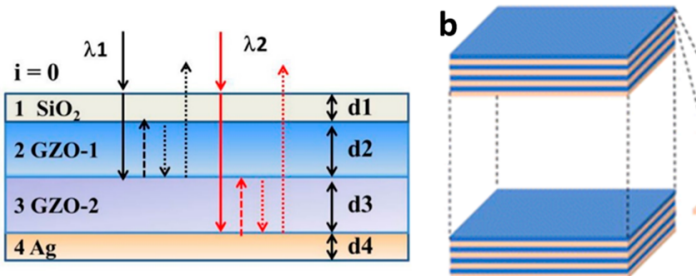

16(AZO-ZnO) multiple layers

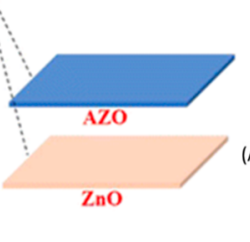

$60 \mathrm{~nm} /$ layer d

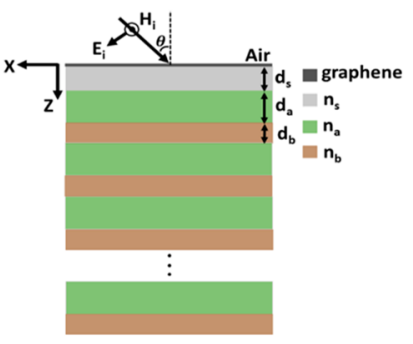

g

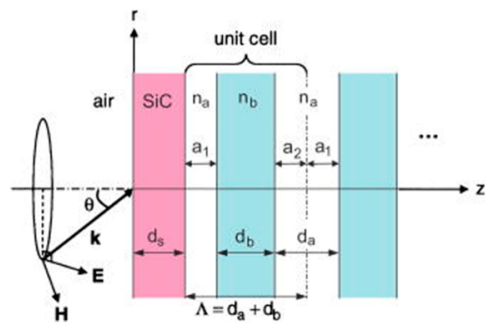

e

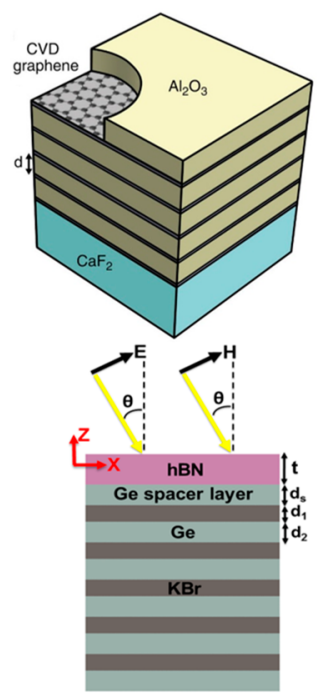

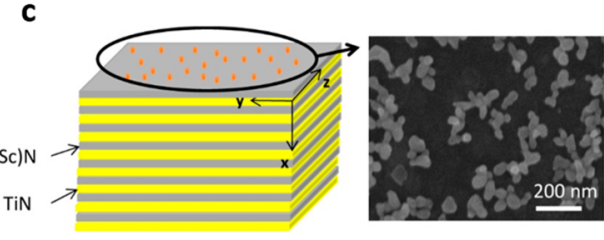

Diso Ne2,200 nm

\section{f}

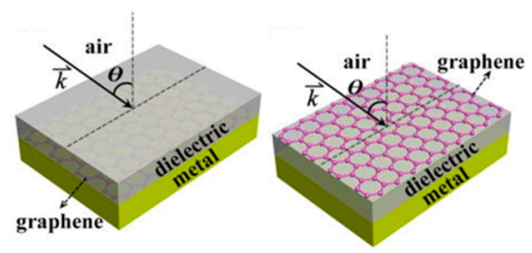

i

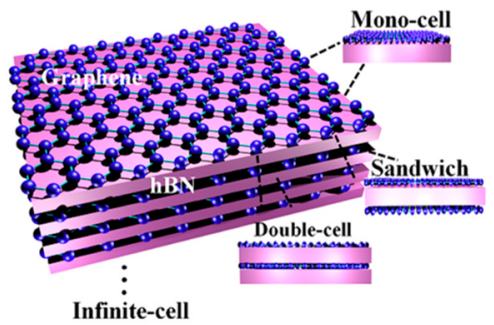

Figure 5. Schematics of different unpatterned plasmonic/phononic light absorbers. (a) An unpatterned $\mathrm{SiO}_{2} /$ bilayer GZO/Ag multilayer structure that acts a broadband perfect absorber within 1.24 to $1.49 \mu \mathrm{m} .{ }^{165}$ (b) Multilayer metamaterial composed of alternating layers of AZO and $\mathrm{ZnO}$ layers that functions as a nearly perfect NIR absorber. ${ }^{167}$ (c) Multilayer metamaterial that is composed of alternating layers of TiN and aluminum scandium nitride $(\mathrm{Al}, \mathrm{Sc}) \mathrm{N}$. The surface of the planar metamaterial is stochastically covered by spin-coated ITO nanoparticles. This metamaterial acts as a light absorber in the NIR region. ${ }^{169}$ (d) A graphene-based 1D PC that can act a narrow-band light absorber in the THz range. ${ }^{176}$ (e) First experimental demonstration of a graphene-dielectric multilayer structure that behaves as a hyperbolic metamaterial in the MIR region. ${ }^{186}$ This structure is capable of light absorption in the MIR and FIR ranges. (f) Unpatterned layered structures in dielectric/graphene/metal and graphene/ dielectric/metal arrangements for UV light absorption. ${ }^{189}(\mathrm{~g})$ SiC-based 1D PC that is appropriate for light absorption within 10.3-12.6 $\mu \mathrm{m}$ window in the mid-IR range. ${ }^{191}(\mathrm{~h}) \mathrm{hBN}$-based 1D PC that can act as nearly perfect light absorption and coherent thermal emission within two 6.2-7.3 $\mu \mathrm{m}$ and $12-12.82 \mu \mathrm{m}$ windows in the MIR range. ${ }^{48}$ (i) Multilayer graphene-hBN metamaterial. ${ }^{192}$ Due to the plasmon-phonon hybridization, this structure has multifunctional capabilities, specially promising for light absorption in a wide window in the MIR range. (a) Reprinted with permission from ref 165. Copyright 2016 IOP Publishing. (b) Reprinted with permission from ref 167. Copyright 2018 Springer Nature. (c) Reprinted with permission from ref 169. Copyright 2014 The Optical Society. (d) Reprinted with permission from ref 176. Copyright 2017 Chinese Laser Press. (e) Reprinted with permission from ref 186. Copyright 2016 Springer Nature. (f) Reprinted with permission from ref 189. Copyright 2018 American Institute of Physics. (g) Reprinted with permission from ref 191. Copyright 2005 American Institute of Physics. (h) Reprinted with permission from ref 48. Copyright 2017 The Optical Society. (i) Reprinted with permission from ref 192. Copyright 2017 American Chemical Society.

further hamper the efficiency of this process, this device configuration has some limitations. Moreover, surface reflection from ultrathin top metal reduces the amount of light absorbed by the semiconductor layer. This configuration can be modified by our proposed design where the spacer layer is replaced by a semiconducting metal oxide such as $\mathrm{TiO}_{2}$ or $\mathrm{SnO}_{2}$. In this configuration, the light is strongly absorbed by top ultrathin photoactive layer. Then, the photogenerated electron is transferred through the metal oxide spacer toward the back metallic contact and the left holes undergo water oxidation reaction. Unlike the previous case, wherein the photoactive layer is a thick coating, such an ultrathin semiconductor layer can ensure the efficient participation of the carrier in the photoelectrochemical reaction even in low mobility semiconductor materials. This design strategy has been successfully applied to $\mathrm{Au} / \mathrm{SnO}_{2} / \mathrm{BiVO}_{4} \mathrm{MIS}$ configuration, where substantial improvement has been obtained by transferring this cavity on top of a prepatterned trapping scaffold, ${ }^{161}$ see Figure $4 \mathrm{f}$.

As mentioned hereinabove, this MIS configuration is not a good solution to get light perfect absorption in dimensions below $1 \mathrm{~nm}$ that corresponds to the thickness of $2 \mathrm{D}$ semiconductor monolayers. That is the reason why MS and MIS based 2D TMD perfect absorbers (absorption above 0.9) have been only realized in multilayer dimensions. ${ }^{154,155}$ The alternative strategy to obtain light perfect absorption in the monolayer of semiconductor layers is to integrate this monolayer with a photonic crystal (PC) design. In one of the pioneer works, authors proved strong light absorption in atomically thin materials through a dielectric cavity design with chirped Bragg reflectors for broadband coherent light absorption. ${ }^{163}$ It was numerically proven that due to this cavity enhancement, a monolayer $\mathrm{MoS}_{2}$ photodetector absorbs as much as $33 \%$ of incident visible light over a $300 \mathrm{~nm}$ bandwidth. Later, another study illustrated narrowband 
absorption above $96 \%$ in a monolayer of $\mathrm{MoS}_{2}$. The structure is made of a $1 \mathrm{D} \mathrm{PC}$ on top of a $\mathrm{MoS}_{2}$ layer, an optically thick reflecting bottom metal mirror, and a spacer between $\mathrm{MoS}_{2}$ and the bottom metal film. ${ }^{164}$ Numerical simulations demonstrated that this strong light absorption is originated from the strong field confinement due to the support of Tamm plasmon modes in the $\mathrm{MoS}_{2}$-based 1D PC. Recently, it was shown that the integration of lossy $2 \mathrm{D}$ materials with a finite all-dielectric 1D random medium can provide random coherent light absorption above $99.9 \%$ in the visible regime, ${ }^{162}$ as shown in Figure 4g. It was found that optical resonances, only in the regime of Anderson localization, can provide the condition for random coherent perfect light absorption.

Other Types of Perfect Absorbers. Alternative plasmonic materials, such as gallium-doped zinc oxide (GZO), aluminum doped zinc oxide (AZO), indium-tin-oxide (ITO), and titanium nitride $(\mathrm{TiN})$, can also be implemented in designing unpatterned metamaterial absorbers. The real part of permittivity of these materials is negative in the NIR region and their epsilon-near-zero (ENZ) characteristics around 1.5 $\mu \mathrm{m}$ can be tuned by doping. ${ }^{140}$ Figure 5a shows schematic of an unpatterned $\mathrm{SiO}_{2}$ /bilayer $\mathrm{GZO} / \mathrm{Ag}$ multilayer structure. ${ }^{165}$ The bilayer GZO is composed of two GZO layers with different carrier concentrations. This structure can act a broadband perfect absorption within 1.24 to $1.49 \mu \mathrm{m}$ and its absorption remained higher than $97 \%$ for incident angles up to $60^{\circ}$. This NIR perfect absorber has a simple design as well as broadband and wide-angle absorption features, which is promising for practical applications. A similar ITO-based structure has also been employed for this purpose. ${ }^{166}$ It has also been verified that a multilayer metamaterial composed of alternating layers of $\mathrm{AZO}$ and $\mathrm{ZnO}$ functions as a nearly perfect NIR absorber (see the schematics in Figure $5 \mathrm{~b}$ that is taken from ref 167). As mentioned in the theoretical background section, this system is another category of unpatterned metamaterial absorbers. In this structure, which does not include any bottom reflecting layer, the negative real part of permittivity of AZO allows the dielectric multilayer to meet the impendence matching condition around $1.90 \mu \mathrm{m}$, leading to almost $99 \%$ light absorption in this region. ${ }^{168}$ As mentioned about, TiN is also another plasmonic material beyond gold and silver. Schematic of a multilayer metamaterial that is composed of alternating layers of $\mathrm{TiN}$ and aluminum scandium nitride $(\mathrm{Al}, \mathrm{Sc}) \mathrm{N}$ is shown in Figure $5 \mathrm{c}{ }^{169}$ As seen from this schematic and the represented SEM image, the surface of the planar metamaterial is stochastically covered by spin-coated ITO nanoparticles on its top. It was reported that the presence of the plasmonic ITO nanoparticles at the surface of the metamaterials facilitates the impedance matching condition by exciting high $-k$ propagating modes in the system and reducing the reflection. Thus, light can penetrate inside the system with minimum reflection and finally absorbed inside the metamaterial.

Other category of the plasmonic materials that can be employed for light absorption in the mid-IR and far-IR regions is $2 \mathrm{D}$ materials such as graphene ${ }^{170-176}$ and black phosphorus. ${ }^{177-183}$ The optical properties of these 2D materials are quite different than those of bulk, 3D materials, which results in significantly different plasmon dispersion relationships. After the rise of graphene, a great deal of attention has been attracted to its potential applications in optoelectronics ${ }^{184}$ and plasmonics. ${ }^{171}$ The surface conductivity of graphene $(\sigma)$ can be effectively modulated via tuning of chemical potential through chemical doping or electrostatic/ magnetostatic gating. ${ }^{170,184,185}$ When $\operatorname{Im}(\sigma)>0$, graphene behaves like a very thin metal layer capable of supporting transverse-magnetic (TM) guided plasmonic mode ${ }^{171-176}$ Tunability of its plasmon resonance through the variation of $\mu$ together with a relatively large propagation length and a small localization length of SPPs in the mid-infrared (MIR), far-IR (FIR) and terahertz (THz) ranges ${ }^{172}$ are the key advantages of the graphene SPPs over those supported by the noble metals. ${ }^{134}$ Consequently, neglecting graphene-based patterned metamaterials, ${ }^{173-175}$ it is possible to obtain nearly perfect resonant absorption in the MIR, FIR, and even $\mathrm{THz}$ frequency ranges by combining an unpatterned layer of graphene with a 1D PC with appropriate design, see the schematics in Figure 5d. ${ }^{176}$ The basic mechanism for the observation of such a resonant behavior in this graphene-based 1D PC is the excitation of Tamm plasmons. Updating eqs 4 and 7 by replacing a single layer of graphene with the optical conductivity $\sigma$ with a lossy film with permittivity $\varepsilon_{\text {lossy }}$ and thickness $t$, we can arrive at the dispersion relations of Tamm plasmons supported by the system. ${ }^{49}$ Taking the structural parameters appropriately, the dispersion curves of the Tamm plasmons at the desired range of frequency can be obtained, and thus, the frequency- and angle-dependency of the resonant absorption peaks of the structure can be precisely estimated. An example of $\mathrm{THz}$ nearly perfect resonant absorptions using a $1 \mathrm{D}$ graphene-based PC is discussed in ref 176 . Recalling that the schematic of this structure is illustrated in panel (d) of Figure 5, the considered $1 \mathrm{D} \mathrm{PC}$ is composed of periodic layers of $\mathrm{SiO}_{2}\left(n_{\mathrm{a}}=1.46\right.$ and $\left.d_{\mathrm{a}}=51.37 \mu \mathrm{m}\right)$ and poly 4-methyl pentene- $1\left(n_{\mathrm{b}}=1.9\right.$ and $\left.d_{\mathrm{b}}=39.47 \mu \mathrm{m}\right)$ and the refractive index and the thickness of the spacer layer are taken to be as 2.12 and $35.35 \mu \mathrm{m}$. It is demonstrated in this paper that the electric field is mostly localized at the dielectric layer below graphene, that is, the plasmonic material, for the Tamm plasmons supported by the plasmonic-based 1D PC. Moreover, the spectral-directional absorption of the structure for TM and TE polarizations verifies that the trend of the nearly perfect absorption behavior of this system is completely predictable by the dispersion of Tamm plasmons. It is noteworthy that, by the same approach, nearly perfect resonant absorption behavior is also achievable by unpatterned layer of black phosphorus. Similar to multilayer metal/dielectric metamaterial, graphene/dielectric metamaterials, that has been realized experimentally (see Figure $5 \mathrm{e}$ that is taken from ref 186), are also another candidate to achieve tunable light absorption in the MIR range. ${ }^{186-188}$ Notice that the designs schematically shown in panels (d) and (e) of Figure 5 are a couple of examples of plasmonic light absorbers based on unpatterned graphene sheet that can operate in the MIR, FIR, and $\mathrm{THz}$ regions. However, graphene, which acts as a lossy dielectric not a plasmonic material, has also potential applications in the Vis and UV ranges. As schematically illustrated in Figure $5 f^{189}$ an unpatterned layer of graphene in dielectric/graphene/metal and graphene/dielectric/metal structures is a decent tool to achieve UV light absorption. It has been reported in ref 189 that by taking the material and structural parameters appropriately, and also by manipulating the polarization and angle of incident light, it is possible to get $71.4 \%$ and $92.2 \%$ light absorption in the planar structures that are composed of single and four layers of graphene, respectively. 


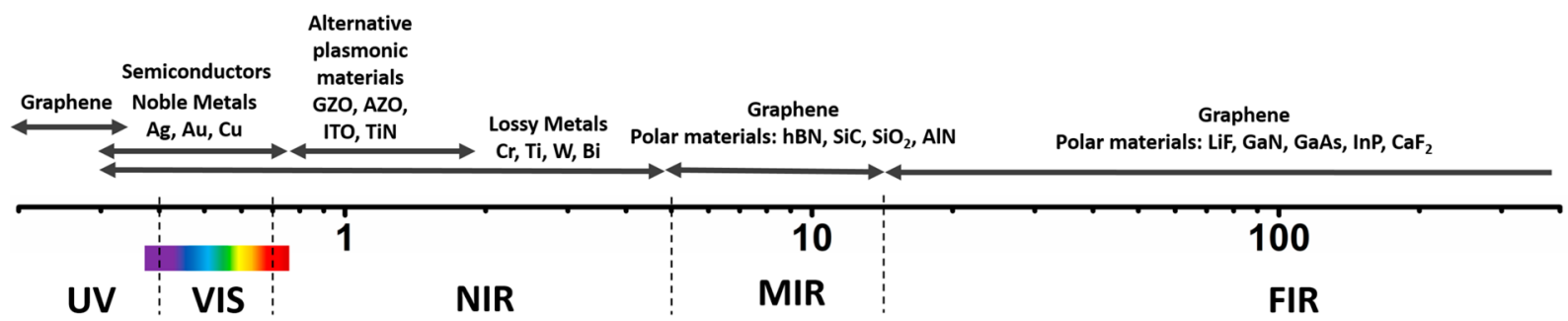

\section{Wavelength $(\mu \mathrm{m})$}

Figure 6. Graph summarizing the proper choice of materials to realize light perfect absorption in a subwavelength, lithography-free, planar, multilayer design.

Alongside the noble metals, highly doped semiconductors, alternative plasmonic materials, graphene and the other $2 \mathrm{D}$ materials, polar dielectrics also offer an opportunity of simultaneous subdiffractional confinement, low optical losses, and operation in the MIR to $\mathrm{THz}$ spectral ranges through the stimulation of surface phonon polariton (SPhP) modes. ${ }^{190}$ $\mathrm{SPhPs}$ originate from the interaction of optical phonons with long-wavelength incident fields, creating a surface excitation mediated by the atomic vibrations. Depending on the type of the polar material, SPhPs have many applications in a wide frequency range from MIR (e.g., hexagonal boron nitride (hBN), ${ }^{193-203} \mathrm{SiC},{ }^{191,204-208} \mathrm{SiO}_{2},{ }^{209}$ and $\mathrm{AlN}^{210}$ ) to FIR (e.g., $\mathrm{LiF}^{211,212} \mathrm{GaN}^{213} \mathrm{GaAs},{ }^{214} \mathrm{InP},{ }^{190}$ and $\mathrm{CaF}_{2}{ }^{190}$ ). Such SPhP modes can be stimulated between the longitudinal optical (LO) and transverse optical (TO) phonon frequencies of the polar dielectrics. This spectral range is referred as the Reststrahlen (RS) band or polaritonic gap. Similar to the metal-based and graphene-based 1D PCs discussed above, SiCbased 1D PC (as a polar-based 1D PC) is also an ${ }^{208}$ approappropriate candidate for nearly perfect light absorption and, thus, coherent thermal emission in 10.3 to $12.6 \mu \mathrm{m}$ in the MIR range. ${ }^{191,208}$ This range is in fact the polaritonic gap of $\mathrm{SiC}$. The schematic of this structure is depicted in Figure $5 \mathrm{~g}$ that is taken from. ${ }^{191}$ It is noteworthy that, according to Kirchhoff's law, the spectral-directional emissivity, is the same as the spectral-directional absorptivity of the system. Notice that coherent thermal emission from surface relief gratings holds promise for spectral and directional control of thermal radiation but is limited to transverse magnetic waves, which can excite surface plasmon or phonon polaritons in the patterned structures. However, for the SiC-based ID PC, due to the support of Tamm phonons, a coherent thermal source can be realized by an unpatterned structure. ${ }^{191,208}$ It should be mentioned that another promising application for these IR absorbing materials is the thermal detection. Thermal detection is currently revolutionizing the IR technology and it is envisioned to expand the market for cameras. These thermal detectors are essentially micro bolometers that are fabricated using micromachining of a thermistor material. Nichrome ( $\mathrm{NiCr}$ ) is one of the most studied $\mathrm{THz}$ absorbing materials used in these designs. ${ }^{215,216}$ Considering the fact that these thermal detectors need no cryogenic cooling, they are promising options for future compact, lightweight, and potentially low-cost cameras.

Another distinguishable polar material suitable for light absorption and thermal emission purposes in the mid-IR region is hBN. As schematically shown in Figure $5 \mathrm{~h}, \mathrm{hBN}$ based 1D PC that is composed on an unpatterned film of hBN and 1D PC separated by a spacer layer, is another candidate for nearly perfect resonant light absorption and coherent thermal emission in the MIR range. ${ }^{48}$ Hexagonal boron nitride is a natural hyperbolic material, for which the dielectric constants are the same in the basal plane $\left(\varepsilon_{\mathrm{t}} \equiv \varepsilon_{x}=\varepsilon_{y}\right)$ but have opposite signs $\left(\varepsilon_{\mathrm{t}} \varepsilon_{z}<0\right)$ in the normal one $\left(\varepsilon_{z}\right)$ in $6.2-7.3 \mu \mathrm{m}$ and $12-$ $12.82 \mu \mathrm{m}$ wavelengths in the mid-IR region, respectively, named as RS-II and RS-I regions. RS-I and RS-II bands represent the wavelength regions at which hyperbolic phonon polaritons (HPPs) supported by hBN show type I and type II hyperbolicities, respectively. Owing to this property, finitethickness slabs of hBN are capable of supporting subdiffractional volume-confined polaritons and can act as multimode waveguides for the propagation of HPP collective modes for TM polarization that originate from the coupling between photons and electric dipoles in phonons. ${ }^{193-203}$ It has been recently reported that, due to the support of HPPs, $1 \mathrm{D}$ grating of hBN is capable of supporting highly concentrated resonant absorptions. ${ }^{198}$ In addition, because of the coupling of magnetic polaritons in metal gratings with HPPs of $h B N$ in TM polarization, MIR perfect or nearly perfect absorption can be observed by an unpatterned film of $\mathrm{hBN}$ on top of a metallic grating. ${ }^{199}$ Moreover, patterned metamaterials that are composed of graphene-hBN heterostructures are also another promising candidate for the support of tunable nearly perfect resonant absorptions. ${ }^{195}$ However, as illustrated in Figure $5 \mathrm{~h}$, the structure under our consideration in the hBN-based 1D PC is composed of unpatterned film of hBN with thickness $t$ and $\varepsilon_{\mathrm{hBN}}$. The 1D PC is composed of alternating layers of $\mathrm{KBr}\left(\varepsilon_{1}\right.$ $\left.=2.25, d_{1}=923 \mathrm{~nm}\right)$ and $\mathrm{Ge}\left(\varepsilon_{2}=16, d_{2}=576 \mathrm{~nm}\right)$ with a spacer layer of $\mathrm{Ge}$ of thickness $d_{s}$. Due to hybridization of surface waves of the 1D PC and HPPs of hBN, Tamm phonons can be supported by the system for both polarizations. As discussed in, ${ }^{48}$ both spectral and directional characteristics of the nearly perfect resonant absorptions of the hBN-based 1D PC can be exactly predicted by dispersion of Tamm modes supported by the structure. Consequently, evaluating Tamm modes by eqs 4 and 7 is a true approach for a reliable estimation of the resonant absorption supported by unpatterned, lossy-based 1D PC. Moreover, mode profiles of the Tamm phonons highlights this point that, in contrast to Tamm plasmons, magnetic field is localized under the polar film for these modes. ${ }^{48}$

As we mentioned earlier, several types of polar materials have been realized to operate in the MIR and FIR ranges. Notice that by using a similar approach, that is, by appropriately designing unpatterned polar-based 1D PCs, it is possible to achieve nearly perfect resonant absorptions at the corresponding wavelengths. As depicted in Figure 5i, multilayer graphene/hBN metamaterial (hypercrystal) is the last 
structure that we would like to investigate as a tunable device appropriate for coherent light absorption and thermal emission in the MIR range. ${ }^{192}$ Through the investigation of the band structure of the metamaterial we found that, due to the coupling between the hybrid plasmon-phonon polaritons supported by each unit cell, the graphene-hBN metamaterial can support hybrid plasmon-phonon polaritons bands. ${ }^{217}$ Moreover, the analysis of light transmission through the metamaterial revealed that this system is capable of supporting high- $k$ propagating hybrid plasmon-phonon polaritons. ${ }^{219}$ Owing to these optical properties, it has been recently reported that this multilayer structure can exceed blackbody thermal radiation in near-field ${ }^{192}$ and also is capable of light absorption in the MIR region. ${ }^{218}$

\section{PERSPECTIVE AND OUTLOOK}

Based on this Perspective, light perfect absorption in the whole EM spectrum (from UV to FIR) can be realized using large scale compatible, planar, lithography-free multilayer designs. For this purpose, it is necessary to find the proper material and design architecture to obtain strong light-matter interaction. Figure 6 outlines the proper materials for different parts of the EM spectrum. In the UV and Vis frequency ranges, this absorption can be obtained by semiconductors and noble metals. Moreover, it was recently proven ${ }^{189}$ that graphene can be also a UV responsive material in a proper planar design. To extend the absorption upper edge toward longer wavelengths such as NIR range, lossy metals such as $\mathrm{Cr}, \mathrm{Ti}$, and $\mathrm{Bi}$ can be a good choice. In the short wavelength NIR regime, alternative plasmonic materials that show epsilon-near-zero (ENZ) characteristic (such as AZO, GZO, and ITO) can be effectively utilized for light near unity absorption. ${ }^{219}$ In these materials, tuning the plasma frequency can provide us the opportunity to control the spectral position of the strong light-matter interaction. Perfect light absorption in longer wavelengths, that is, MIR, FIR, and $\mathrm{THz}$, which are inaccessible with unpatterned metal-based multilayer designs, can be acquired using graphene, black phosphorus, and suitable polar materials, as shown in the figure. Therefore, it can be envisioned that planar light perfect absorbers that do not require sophisticated lithography fabrication techniques, can be an excellent option for future large scale optoelectronic application. For this aim, according to the operation wavelength and the application, the proper design architecture and material should be used.

Metal-based planar metamaterial absorbers can be listed in two main categories; narrowband and broadband perfect absorbers. As illustrated earlier, narrowband perfect light absorbers can be acquired using noble low loss metals such as $\mathrm{Au}$ and Ag. These structures can be of particular interest in color filtering, imaging, and display applications. The planar and large scale compatible nature of these multilayer designs facilitates their integration into commercialized color-CCD/ cameras. ${ }^{87}$ Different from nanostructured plasmonic designs, these Febry-Perot based resonators can operate in a wide incident light polarizations and angles that makes them suitable for practical applications. Moreover, the light absorption can be also obtained in broad/ultrabroad wavelengths. An ideal blackbody absorber that suppresses incident light reflection and transmission in a broad spectral range can ensure efficient use of the solar power. One of the most studied areas of interest for these ultrabroadband perfect absorbers is thermophotovoltaics (TPV). This system harvests the incident photons in a broad spectral range and transduces them into thermal energy and then a frequency-selective emitter, which is matched into the band gap of the PV cell, radiates a narrowband thermal emission. Based on previous works, this system can ideally overcome the fundamental limits of conventional PV cells. ${ }^{20}$ In a PV cell, the absorption is restricted into the photons with energies above the optical band gap of the active layer. Therefore, only a specific portion of the solar spectrum will be efficiently harvested. However, metals can retain their perfect absorption property over the entire Vis and NIR regimes. Covering the most parts of solar irradiation power can guarantee the efficient harvesting of the incident light. Therefore, the use of lossy metals in metalinsulator pair based configuration can provide near unity absorption of the light and this intense absorbed power can be directed into a PV cell using a narrowband emitter to substantiate the PV cell efficiency. The narrowband emitter can be also realized using dielectric based PC structure which makes the whole TPV system lithography-free. ${ }^{104}$ Thus, the integration of a PC narrowband emitter with a refractory lossy metal based ultrabroadband perfect absorber can be considered as a winning strategy for the design of future highly efficient TPV systems. Another area of usage of these ultrabroadband absorbers is solar driven steam generation. Advancements in this area have proved efficiencies as high as $90 \%$ at only 4 -sun intensity $\left(4 \mathrm{~kW} \mathrm{~m}^{-2}\right){ }^{33}$ Therefore, same as TPV, the realization of ultrabroadband light perfect absorbers can be of great interest in the steam generation.

Strong light absorption in ultrathin semiconductor based perfect absorbers can also have high potentials in PV, photodetectors and photoelectrochemical applications. Our theoretical findings revealed that spectrally broad strong lightmatter interaction can be acquired in MIS cavity based designs. Therefore, the incident light is efficiently harvested in subwavelength geometries and the ultrathin thickness of the semiconductor would minimize the transport length of photogenerated carriers that in turn maximizes their collection efficiency. However, the main challenge in these semiconductor-based metamaterials is the crystalline quality of the photoactive layer. Utilizing common deposition and growth techniques for ultrathin films, it is difficult to fabricate single crystalline semiconductor in such thicknesses. In most of these designs, the active layer is made of amorphous semiconductor, and therefore, their optoelectronic performance is far inferior to those of bulk crystalline counterparts. Therefore, the main challenge that limits the replacement of conventional bulk semiconductor-based optoelectronic system with these ultrathin metamaterials is their poor electrical properties of the photoactive layer. A recent study demonstrated a high performance MIS configuration-based photodetector where the single-crystalline $\mathrm{Ge}$ membrane undergoes a strong light-matter interaction on a functionalized nanocavity design. ${ }^{157}$ The use of chemical synthesis methods can be also an alternative to realize single crystalline semiconductors in such nanometer scale thicknesses. Two-dimensional (2D) TMDs are another category of semiconductors that can be employed in ultrathin optoelectronics. Transition from bulk to monolayer makes these semiconductors a direct band gap material and consequently a better absorption dynamics are emerged. However, the atomic thickness of the layer can absorb only a small portion of the light and thus, a trapping mechanism is required to provide strong light-matter interaction. As shown in the above sections, the use of MS or MIS cavity architectures could not be a good solution for 
light perfect absorption for 2D TMDs. These cavity designs can efficiently harvest the light in multiple layer thicknesses. To realize perfect light absorption in the unpatterned monolayer of TMDs, PC configuration is the right strategy to confine the entire power in the position of the monolayer where this confined energy is harvested efficiently. A recent Perspective in the application of these van der Waals materials in PV technology predicted that it is possible, in principle, to achieve power conversion efficiencies as high as $25 \% .{ }^{221}$ Introducing a tandem scheme where these $2 \mathrm{D}$ materials are brought to a junction with another bulk semiconductor (such as $\mathrm{Si}$ ) could further substantiate the cell efficiency. This bulk semiconductor can be replaced with a MIS nanocavity design to localize the absorbed power in ultrathin dimensions. Then, a PC-capped 2D TMD can be grown on top of this design to make an ultrathin efficient tandem solar cell. Therefore, the findings of this study can serve as a beacon for future performance enhanced semiconductor optoelectronic devices.

As mentioned herein above, graphene, black phosphorus, and polar materials are appropriate candidates for achieving light absorption in the MIR, FIR, and $\mathrm{THz}$ ranges. MIR/FIR directional light emitters are a distinguished category of light absorbers that have attracted intense interests during the previous years due to their potential application in radiative heat transfer and energy conversion systems. ${ }^{192,205,207}$ A thermal light-emitting source, such as a blackbody or the incandescent filament of a light bulb, is often presented as a typical example of an incoherent source and is in marked contrast to a laser. Whereas a laser is highly monochromatic and very directional, a thermal source has a broad spectrum and is usually quasi-isotropic. It has been shown recently that the light absorption by polar-based 1D PCs is considerably enhanced in a directional manner compared to a bare polar film. This system, therefore, is capable of emitting radiation efficiently in well-defined directions and can act as a coherent thermal source at a fair distance. For these structures, narrow angular emission lobes similar to antenna lobes are observed and the emission spectra of the source depend on the observation angle. As mentioned earlier, the origin of the coherent emission is in the support of Tamm phonon polaritons by the above-mentioned polar-based ID PC. The unpatterned lossy-based narrowband absorbers may also be employed as radiative coolers that are reflective in the solar spectrum and emissive in the transparency window of the atmosphere $(8-13 \mu \mathrm{m})$. These absorbers, therefore, can be regarded as a critical component in the MIR photodetectors and imaging systems that are important in applications such as night vision and astronomy research. ${ }^{206,222,223}$ MIR/FIR filtering and sensing for detecting gases as well as biological and chemical agents can be mentioned as another potential application of the narrow band light absorbers discussed in this Perspective. $^{224}$

\section{AUTHOR INFORMATION}

\section{Corresponding Authors}

*E-mail: amir@ee.bilkent.edu.tr.

*E-mail: ozbay@bilkent.edu.tr.

\section{ORCID $\odot$}

Amir Ghobadi: 0000-0002-8146-0361

Hodjat Hajian: 0000-0001-6564-6273

\section{Author Contributions}

All of the authors contributed in the writing and discussion of the Perspective.

\section{Notes}

The authors declare no competing financial interest.

\section{ACKNOWLEDGMENTS}

Authors acknowledge financial support from Scientific and Technological Research Council of Turkey (TUBITAK) and DPT-HAMIT under Project Nos. 113E331, 114E374, and 115F560. One of the authors (E.O.) also acknowledges partial support from the Turkish Academy of Sciences (TUBA).

\section{REFERENCES}

(1) Smith, D. R.; Pendry, J. B.; Wiltshire, M. C. K. Metamaterials and Negative Refractive Index. Science 2004, 305, 788-793.

(2) Yao, J.; Liu, Z.; Liu, Y.; Wang, Y.; Sun, C.; Bartal, G.; Stacy, A. M.; Zhang, X. Optical Negative Refraction in Bulk Metamaterials of Nanowires. Science (Washington, DC, U. S.) 2008, 321, 930.

(3) Hoffman, A. J.; Alekseyev, L.; Howard, S. S.; Franz, K. J.; Wasserman, D. A. N.; Podolskiy, V. A.; Narimanov, E. E.; Sivco, D. L.; Gmachl, C. Negative Refraction in Semiconductor Metamaterials. Nat. Mater. 2007, 6, 946-950.

(4) Lapine, M.; Shadrivov, I. V.; Powell, D. A.; Kivshar, Y. S. Magnetoelastic Metamaterials. Nat. Mater. 2012, 11 (1), 30-33.

(5) Fleischman, D.; Davoyan, A.; Yeh, P.; Atwater, H. A. Optical Magnetism in Planar Metamaterial Heterostructures. Nat. Commun. 2018, 9, 296

(6) Menzel, C.; Helgert, C.; Rockstuhl, C.; Kley, E. B.; Tunnermann, A.; Pertsch, T.; Lederer, F. Asymmetric Transmission of Linearly Polarized Light at Optical Metamaterials. Phys. Rev. Lett. 2010, 104 (25), 253902

(7) Li, Z.; Mutlu, M.; Ozbay, E. Chiral Metamaterials: From Optical Activity and Negative Refractive Index to Asymmetric Transmission. J. Opt. 2013, 15, 023001.

(8) Fang, A.; Koschny, T.; Soukoulis, C. M. Lasing in Metamaterial Nanostructures. J. Opt. 2010, 12, 024013.

(9) Plum, E.; Fedotov, V. A.; Kuo, P.; Tsai, D. P.; Zheludev, N. I. Towards the Lasing Spaser: Controlling Metamaterial Optical Response with Semiconductor Quantum Dots. Opt. Express 2009, 17 (10), 11-14.

(10) Schurig, D.; Mock, J. J.; Justice, B. J.; Cummer, S. A.; Pendry, J. B.; Starr, A. F.; Smith, D. R. Metamaterial Electromagnetic Cloak at Microwave Frequencies. Science (Washington, DC, U. S.) 2006, 314, 977-981.

(11) Valentine, J.; Li, J.; Zentgraf, T.; Bartal, G.; Zhang, X. An Optical Cloak Made of Dielectrics. Nat. Mater. 2009, 8 (7), 568-571.

(12) Ergin, T.; Stenger, N.; Brenner, P.; Pendry, J. B.; Wegener, M. Three-Dimensional Invisibility Cloak at Optical Wavelengths. Science (Washington, DC, U. S.) 2010, 328 (5976), 337-339.

(13) Landy, N. I.; Sajuyigbe, S.; Mock, J. J.; Smith, D. R.; Padilla, W. J. Perfect Metamaterial Absorber. Phys. Rev. Lett. 2008, 100, 207402.

(14) Stewart, M. E.; Anderton, C. R.; Thompson, L. B.; Maria, J.; Gray, S. K.; Rogers, J. A.; Nuzzo, R. G. Nanostructured Plasmonic Sensors. Chem. Rev. 2008, 108, 494-521.

(15) Offermans, P.; Schaafsma, M. C.; Rodriguez, S. R. K.; Zhang, Y.; Crego-calama, M. Universal Scaling of the Figure of Merit of Plasmonic Sensors. ACS Nano 2011, 5 (6), 5151-5157.

(16) Xu, T.; Wu, Y.-K.; Luo, X.; Guo, L. J. Plasmonic Nanoresonators for High-Resolution Colour Filtering and Spectral Imaging. Nat. Commun. 2010, 1, 59.

(17) Landy, N. I.; Bingham, C. M.; Tyler, T.; Jokerst, N.; Smith, D. R.; Padilla, W. J. Design Theory, and Measurement of a PolarizationInsensitive Absorber for Terahertz Imaging. Phys. Rev. B: Condens. Matter Mater. Phys. 2009, 79 (12), 1-6.

(18) Tittl, A.; Michel, A. U.; Schäferling, M.; Yin, X.; Gholipour, B.; Cui, L.; Wuttig, M.; Taubner, T.; Neubrech, F.; Giessen, H. A 
Switchable Mid-Infrared Plasmonic Perfect Absorber with Multispectral Thermal Imaging Capability. Adv. Mater. 2015, 27, 45974603.

(19) Ellenbogen, T.; Seo, K.; Crozier, K. B. Chromatic Plasmonic Polarizers for Active Visible Color Filtering and Polarimetry. Nano Lett. 2012, 12, 1026-1031.

(20) Yokogawa, S.; Burgos, S. P.; Atwater, H. A. Plasmonic Color Filters for CMOS Image Sensor Applications. Nano Lett. 2012, 12 (8), 4349-4354.

(21) Ji, C.; Lee, K. T.; Xu, T.; Zhou, J.; Park, H. J.; Guo, L. J. Engineering Light at the Nanoscale: Structural Color Filters and Broadband Perfect Absorbers. Adv. Opt. Mater. 2017, 5 (20), 1700368.

(22) Rephaeli, E.; Fan, S. Absorber and Emitter for Solar ThermoPhotovoltaic Systems to Achieve Efficiency Exceeding the ShockleyQueisser Limit. Opt. Express 2009, 17 (17), 15145-15159.

(23) Tong, J. K.; Hsu, W.; Huang, Y.; Boriskina, S. V.; Chen, G. Thin-Film "Thermal Well" Emitters and Absorbers for HighEfficiency Thermophotovoltaics. Sci. Rep. 2015, 5, 10661.

(24) Wu, C.; Iii, B. N.; John, J.; Milder, A.; Zollars, B.; Savoy, S.; Shvets, G. Metamaterial-Based Integrated Plasmonic Absorber/ Emitter for Solar Thermo-Photovoltaic Systems. J. Opt. 2012, 14, 024005.

(25) Liu, T. J.; Takahara, J. Ultrabroadband Absorber Based on Single- Sized Embedded Metal-Dielectric-Metal Structures and Application of Radiative Cooling. Opt. Express 2017, 25 (12), 1443-1447.

(26) Wu, D.; Liu, C.; Xu, Z.; Liu, Y.; Yu, Z.; Yu, L.; Chen, L.; Li, R. The Design of Ultra-Broadband Selective near-Perfect Absorber Based on Photonic Structures to Achieve near-Ideal Daytime Radiative Cooling. Mater. Des. 2018, 139, 104-111.

(27) Rephaeli, E.; Raman, A.; Fan, S. Ultrabroadband Photonic Structures To Achieve High-Performance Daytime Radiative Cooling. Nano Lett. 2013, 13, 1457-1461.

(28) de Arquer, F. P. G.; Mihi, A.; Konstantatos, G. Large-Area Plasmonic-Crystal-Hot-Electron-Based Photodetectors. ACS Photonics 2015, 2, 950-957.

(29) Li, W.; Valentine, J. Metamaterial Perfect Absorber Based Hot Electron Photodetection. Nano Lett. 2014, 14, 3510-3514.

(30) Ghobadi, T. G. U.; Ghobadi, A.; Ozbay, E.; Karadas, F. Strategies for Plasmonic Hot-Electron-Driven Photoelectrochemical Water Splitting. ChemPhotoChem. 2018, 2, 1-23.

(31) Fang, J.; Liu, Q.; Zhang, W.; Gu, J.; Su, Y.; Su, H.; Guo, C.; Zhang, D. Ag/Diatomite for Highly Efficient Solar Vapor Generation under One-Sun Irradiation. J. Mater. Chem. A 2017, 5, 17817-17821.

(32) Bae, K.; Kang, G.; Cho, S. K.; Park, W.; Padilla, W. J.; Kim, K. Flexible Thin-Film Black Gold Membranes with Ultrabroadband Plasmonic Nanofocusing for Efficient Solar Vapour Generation. Nat. Commun. 2015, 6, 1-9.

(33) Zhou, L.; Tan, Y.; Ji, D.; Zhu, B.; Zhang, P.; Xu, J.; Gan, Q.; Yu, Z.; Zhu, J. Self-Assembly of Highly Efficient, Broadband Plasmonic Absorbers for Solar Steam Generation. Sci. Adv. 2016, 2 (4), e1501227-e1501227.

(34) Park, J.; Kang, J.; Vasudev, A. P.; Schoen, D. T.; Kim, H.; Hasman, E.; Brongersma, M. L. Omnidirectional Near-Unity Absorption in an Ultrathin Planar Semiconductor Layer on a Metal Substrate. ACS Photonics 2014, 1, 812-821.

(35) Hägglund, C.; Apell, S. P. Plasmonic Near-Field Absorbers for Ultrathin Solar Cells. J. Phys. Chem. Lett. 2012, 3, 1275-1285.

(36) Detectors, P.; Entezari, M.; Zavvari, M. Application of Hyperbolic Metamaterials for Responsivity Enhancement of Thin Film Photo-Conductive Detectors. IEEE Sens. J. 2016, 16 (24), $8916-8920$

(37) Wang, W.; Klots, A.; Prasai, D.; Yang, Y.; Bolotin, K. I.; Valentine, J. Hot Electron-Based Near-Infrared Photodetection Using Bilayer MoS 2. Nano Lett. 2015, 15, 7440-7444.

(38) Ferry, V. E.; Verschuuren, M. A.; Li, H. B. T.; Verhagen, E.; Walters, R. J.; Schropp, R. E. I.; Atwater, H. A.; Polman, A. Light
Trapping in Ultrathin Plasmonic Solar Cells. Opt. Express 2010, 18, 237-245.

(39) Lopez-Sanchez, O.; Alarcon Llado, E.; Koman, V.; Fontcuberta I Morral, A.; Radenovic, A.; Kis, A. Light Generation and Harvesting in a van Der Waals Heterostructure. ACS Nano 2014, 8 (3), 30423048.

(40) Spinelli, P.; Ferry, V. E.; Van De Groep, J.; Van Lare, M.; Verschuuren, M. A.; Schropp, R. E. I.; Atwater, H. A.; Polman, A. Plasmonic Light Trapping in Thin-Film Si Solar Cells. J. Opt. 2012, 14, 024002.

(41) Wang, Y.; Sun, T.; Paudel, T.; Zhang, Y.; Ren, Z.; Kempa, K. Metamaterial-Plasmonic Absorber Structure for High Efficiency Amorphous Silicon Solar Cells. Nano Lett. 2012, 12, 440-445.

(42) Green, M. A.; Pillai, S. Harnessing Plasmonics for Solar Cells. Nat. Photonics 2012, 6, 130-132.

(43) Vandamme, N.; Bardou, N.; Dupuis, C. Metal Nanogrid for Broadband Multiresonant Light-Harvesting in Ultrathin GaAs Layers. ACS Photonics 2014, 1, 878-884.

(44) Guo, C. F.; Sun, T.; Cao, F.; Liu, Q.; Ren, Z. Metallic Nanostructures for Light Trapping in Energy-Harvesting Devices. Light: Sci. Appl. 2014, 42, 1-12.

(45) Ahmadivand, A.; Karabiyik, M.; Pala, N. 6. Plasmonic Photodetectors; Elsevier Ltd, 2016.

(46) Ghobadi, A.; Hajian, H.; Rashed, A. R.; Butun, B.; Ozbay, E. Tuning the Metal Filling Fraction in Absorbers to Maximize the Absorption Bandwidth. Photonics Res. 2018, 6 (3), 1-9.

(47) Steslicka, M.; Kucharczyk, R.; Akjouj, A.; Djafari-rouhani, B.; Dobrzynski, L.; Davison, S. G. Localised Electronic States in Semiconductor Superlattices. Surf. Sci. Rep. 2002, 47, 93-196.

(48) Hajian, H.; Ghobadi, A.; Butun, B.; Ozbay, E. Nearly Perfect Resonant Absorption and Coherent Thermal Emission by HBNBased Photonic Crystals. Opt. Express 2017, 25 (25), na.

(49) Hajian, H.; Soltani-vala, A.; Kalafi, M. Optimizing Terahertz Surface Plasmons of a Monolayer Graphene and a Graphene Parallel Plate Waveguide Using One-Dimensional Photonic Crystal. J. Appl. Phys. 2013, 114, 033102.

(50) Ogawa, S.; Kimata, M. Metal-Insulator-Metal-Based Plasmonic Metamaterial Absorbers at Visible and Infrared Wavelengths: A Review. Materials 2018, 11 (458), 1-18.

(51) Liu, N.; Mesch, M.; Weiss, T.; Hentschel, M.; Giessen, H. Infrared Perfect Absorber and Its Application as Plasmonic Sensor. Nano Lett. 2010, 10 (7), 2342-2348.

(52) Aydin, K.; Ferry, V. E.; Briggs, R. M.; Atwater, H. a. Broadband Polarization-Independent Resonant Light Absorption Using Ultrathin Plasmonic Super Absorbers. Nat. Commun. 2011, 2, 517.

(53) Hao, J.; Wang, J.; Liu, X.; Padilla, W. J.; Zhou, L.; Qiu, M. High Performance Optical Absorber Based on a Plasmonic Metamaterial. Appl. Phys. Lett. 2010, 96, 251104.

(54) Hedayati, M. K.; Javaherirahim, M.; Mozooni, B.; Abdelaziz, R.; Tavassolizadeh, A.; Chakravadhanula, V. S. K.; Zaporojtchenko, V.; Strunkus, T.; Faupel, F.; Elbahri, M. Design of a Perfect Black Absorber at Visible Frequencies Using Plasmonic Metamaterials. Adv. Mater. 2011, 23 (45), 5410-5414.

(55) Hao, J.; Zhou, L.; Qiu, M. Nearly Total Absorption of Light and Heat Generation by Plasmonic Metamaterials. Phys. Rev. B: Condens. Matter Mater. Phys. 2011, 83, 165107.

(56) Watts, C. M.; Liu, X.; Padilla, W. J. Metamaterial Electromagnetic Wave Absorbers. Adv. Mater. 2012, 24, OP98-OP120.

(57) Wen, Q.; Xie, Y.; Zhang, H.; Yang, Q.; Li, Y. Transmission Line Model and Fields Analysis of Metamaterial Absorber in the Terahertz Band. Opt. Express 2009, 17 (22), 20256-20265.

(58) Yan, M. Metal-Insulator-Metal Light Absorber: A Continuous Structure. J. Opt. 2013, 15 (2), 25006.

(59) Tao, H.; Bingham, C. M.; Pilon, D.; Fan, K.; Strikwerda, A. C.; Shrekenhamer, D.; Padilla, W. J.; Zhang, X.; Averitt, R. D. A Dual Band Terahertz Metamaterial Absorber. J. Phys. D: Appl. Phys. 2010, 43,225102 
(60) Yong, Z.; Zhang, S.; Gong, C.; He, S. Narrow Band Perfect Absorber for Maximum Localized Magnetic and Electric Field Enhancement and Sensing Applications. Sci. Rep. 2016, 6, 24063.

(61) Mandal, P. Plasmonic Metamaterial Perfect Absorber at Visible Frequency: Design Rule. J. Nanophotonics 2017, 11 (3), 036003.

(62) Wang, B.; Huang, W.-Q.; Wang, L.-L. Ultra-Narrow Terahertz Perfect Light Absorber Based on Surface Lattice Resonance of a Sandwich Resonator for Sensing Applications. RSC Adv. 2017, 7, $42956-42963$.

(63) Wu, D.; Liu, Y.; Li, R.; Chen, L.; Ma, R.; Liu, C.; Ye, H. Infrared Perfect Ultra-Narrow Band Absorber as Plasmonic Sensor. Nanoscale Res. Lett. 2016, 11 (1), 483.

(64) Kim, J.; Han, K.; Hahn, J. W. Selective Dual-Band Metamaterial Perfect Absorber for Infrared Stealth Technology. Sci. Rep. 2017, 7, 6740.

(65) Shu, S.; Li, Z.; Li, Y. Y. Triple-Layer Fabry-Perot Absorber with near- Perfect Absorption in Visible and near-Infrared Regime. Opt. Express 2013, 21 (21), 4795-4801.

(66) Do, Y. S.; Choi, K. C. Poly-Periodic Hole Arrays for AngleInvariant Plasmonic Filters. Opt. Lett. 2015, 40 (16), 3873-3876.

(67) Moreau, A.; Cirac1, C.; Mock, J. J.; Hill, R. T.; Wang, Q.; Wiley, B. J.; Chilkoti, A.; Moreau, A.; Cirac1, C.; Smith, D. R. ControlledReflectance Surfaces with Film-Coupled Colloidal Nanoantennas. Nature 2012, 492, 86-90.

(68) Cattoni, A.; Ghenuche, P.; Decanini, D.; Chen, J.; Pelouard, J. $\Lambda 3 / 1000$ Plasmonic Nanocavities for Biosensing Fabricated by Soft UV Nanoimprint Lithography. Nano Lett. 2011, 11, 3557-3563.

(69) Zhang, N.; Dong, Z.; Ji, D.; Song, H.; Zeng, X.; Liu, Z.; Jiang, S.; Xu, Y.; Bernussi, A. Reversibly Tunable Coupled and Decoupled Super Absorbing Structures. Appl. Phys. Lett. 2016, 108, 091105.

(70) Chang, W.; Won, J.; Slaughter, L. S.; Link, S. Plasmonic Nanorod Absorbers as Orientation Sensors. Proc. Natl. Acad. Sci. U. S. A. 2010, 107 (7), 2781-2786.

(71) Avitzour, Y.; Urzhumov, Y. A.; Shvets, G. Wide-Angle Infrared Absorber Based on a Negative-Index Plasmonic Metamaterial. Phys. Rev. B: Condens. Matter Mater. Phys. 2009, 79 (4), 1-5.

(72) Liu, X.; Starr, T.; Starr, A. F.; Padilla, W. J. Infrared Spatial and Frequency Selective Metamaterial with Near-Unity Absorbance. Phys. Rev. Lett. 2010, 104, 207403.

(73) Wang, H.; Wang, L. Perfect Selective Metamaterial Solar Absorbers. Opt. Express 2013, 21 (S6), 13311-13319.

(74) Nielsen, M. G.; Pors, A.; Albrektsen, O.; Bozhevolnyi, S. I. Efficient Absorption of Visible Radiation by Gap Plasmon Resonators. Opt. Express 2012, 20 (12), 13311-13319.

(75) Ming, X.; Tan, Q. Design Method of a Broadband Wide-Angle Plasmonic Absorber in the Visible Range. Plasmonics 2017, 12 (1), 14-17.

(76) Kenney, M.; Grant, J.; Shah, Y. D.; Escorcia-carranza, I.; Humphreys, M.; Cumming, D. R. S. Octave-Spanning Broadband Absorption of Terahertz Light Using Metasurface Fractal-Cross Absorbers. ACS Photonics 2017, 4, 2604-2612.

(77) Duan, X.; Chen, S.; Liu, W.; Cheng, H.; Li, Z. PolarizationInsensitive and Wide-Angle Broadband Nearly Perfect Absorber by Tunable Planar Metamaterials in the Visible Regime. J. Opt. 2014, 16, 125107.

(78) Azad, A. K.; Kort-kamp, W. J. M.; Sykora, M.; Weisse-bernstein, N. R.; Luk, T. S.; Taylor, A. J.; Dalvit, D. A. R.; Chen, H. Metasurface Broadband Solar Absorber. Sci. Rep. 2016, 6, 20347.

(79) Mudachathi, R.; Tanaka, T. Broadband Plasmonic Perfect Light Absorber in the Visible Spectrum for Solar Cell Applications. Adv. Nat. Sci.: Nanosci. Nanotechnol. 2018, 9, 015010.

(80) Lu, Y.; Dong, W.; Chen, Z.; Pors, A.; Wang, Z.; Bozhevolnyi, S. I. Gap-Plasmon Based Broadband Absorbers for Enhanced HotElectron and Photocurrent Generation. Sci. Rep. 2016, 6, 30650.

(81) Tagliabue, G.; Eghlidi, H.; Poulikakos, D. Facile Multifunctional Plasmonic Sunlight Harvesting with Tapered Triangle Nanopatterning of Thin Films. Nanoscale 2013, 5 (20), 9957-9962.

(82) Ghobadi, A.; Hajian, H.; Gokbayrak, M.; Dereshgi, S. A.; Toprak, A.; Butun, B.; Ozbay, E. Visible Light Nearly Perfect
Absorber: An Optimum Unit Cell Arrangement for near Absolute Polarization Insensitivity. Opt. Express 2017, 25 (22), 27624.

(83) Ji, T.; Wang, Y.; Cui, Y.; Lin, Y.; Hao, Y.; Li, D. Flexible Broadband Plasmonic Absorber on Moth-Eye Substrate. Mater. Today Energy 2017, 5, 181-186.

(84) Bossard, J. A.; Lin, L.; Yun, S.; Liu, L.; Werner, D. H.; Mayer, T. S. Near-Ideal Optical Metamaterial Absorbers with Super-Octave Bandwidth. ACS Nano 2014, 8 (2), 1517-1524.

(85) Ding, F.; Dai, J.; Chen, Y.; Zhu, J.; Jin, Y.; Bozhevolnyi, S. I. Broadband Near-Infrared Metamaterial Absorbers Utilizing Highly Lossy Metals. Sci. Rep. 2016, 6, 39445.

(86) Park, C. S.; Shrestha, V. R.; Lee, S. S.; Choi, D. Y. TransReflective Color Filters Based on a Phase Compensated Etalon Enabling Adjustable Color Saturation. Sci. Rep. 2016, 6, 25496.

(87) Li, Z.; Butun, S.; Aydin, K. Large-Area, Lithography-Free Super Absorbers and Color Filters at Visible Frequencies Using Ultrathin Metallic Films. ACS Photonics 2015, 2 (2), 183-188.

(88) Zhao, Y.; Zhao, Y.; Hu, S.; Lv, J.; Ying, Y.; Gervinskas, G.; Si, G. Artificial Structural Color Pixels: A Review. Materials 2017, 10, 944.

(89) Yoon, Y.-T.; Lee, S.-S. Transmission Type Color Filter Incorporating a Silver Film Based Etalon. Opt. Express 2010, 18 (5), 5344-5349.

(90) Zhao, D.; Meng, L.; Gong, H.; Chen, X.; Chen, Y.; Yan, M.; Li, Q.; Qiu, M. Ultra-Narrow-Band Light Dissipation by a Stack of Lamellar Silver and Alumina. Appl. Phys. Lett. 2014, 104, 221107.

(91) Lee, J. Y.; Lee, K. T.; Seo, S.; Guo, L. J. Decorative Power Generating Panels Creating Angle Insensitive Transmissive Colors. Sci. Rep. 2015, 4, 4192.

(92) Mirshafieyan, S. S.; Gregory, D. A. Electrically Tunable Perfect Light Absorbers as Color Filters and Modulators. Sci. Rep. 2018, 8 (January), 1-9.

(93) Peng, H.; Luo, Y.; Ying, X.; Pu, Y.; Jiang, Y.; Xu, J.; Liu, Z. Broadband and Highly Absorbing Multilayer Structure in MidInfrared. Appl. Opt. 2016, 55 (31), 8833-8838.

(94) Li, Z.; Palacios, E.; Butun, S.; Kocer, H.; Aydin, K. Omnidirectional, Broadband Light Absorption Using Large-Area, Ultrathin Lossy Metallic Film Coatings. Sci. Rep. 2015, 5, 15137.

(95) Ghobadi, A.; Dereshgi, S. A.; Butun, B.; Ozbay, E. UltraBroadband Asymmetric Light Transmission and Absorption Through The Use of Metal Free Multilayer Capped Dielectric Microsphere Resonator. Sci. Rep. 2017, 7, 14538.

(96) Mattiucci, N.; Bloemer, M. J.; Akozbek, N.; D’Aguanno, G. Impedance Matched Thin Metamaterials Make Metals Absorbing. Sci. Rep. 2013, 3 (3203), 1-11.

(97) Chirumamilla, M.; Roberts, A. S.; Ding, F.; Wang, D.; Kristensen, P. K.; Bozhevolnyi, S. I.; Pedersen, K. Multilayer Tungsten-Alumina-Based Broadband Light Absorbers for HighTemperature Applications. Opt. Mater. Express 2016, 6 (8), 2704.

(98) Mao, K.; Shen, W.; Yang, C.; Fang, X.; Yuan, W.; Zhang, Y.; Liu, X. Angle Insensitive Color Filters in Transmission Covering the Visible Region. Sci. Rep. 2016, 6, 19289.

(99) Deng, H.; Li, Z.; Stan, L.; Rosenmann, D.; Czaplewski, D. Broadband Perfect Absorber Based on One Ultrathin Layer of Refractory Metal. Opt. Lett. 2015, 40 (11), 2592-2595.

(100) Kajtár, G.; Kafesaki, M.; Economou, E. N.; Soukoulis, C. M. Theoretical Model of Homogeneous Metal-insulator-metal Perfect Multi-Band Absorbers for the Visible Spectrum. J. Phys. D: Appl. Phys. 2016, 49 (5), 055104.

(101) Ding, F.; Mo, L.; Zhu, J.; He, S.; Ding, F.; Mo, L.; Zhu, J.; He, S. Lithography-Free, Broadband, Omnidirectional, and PolarizationInsensitive Thin Optical Absorber. Appl. Phys. Lett. 2015, 106, 061108.

(102) Zhong, Y. K.; Lai, Y.-C.; Tu, M.-H.; Chen, B.-R.; Fu, S. M.; Yu, P.; Lin, A. Omnidirectional, Polarization-Independent, UltraBroadband Metamaterial Perfect Absorber Using Field-Penetration and Reflected-Wave-Cancellation. Opt. Express 2016, 24 (10), A832.

(103) Zhong, Y. K.; Tu, M.; Chen, B.; Lin, A.; Nanostructures, P.; Zhong, Y. K.; Fu, S. M.; Ju, N. P.; Tu, M.; Chen, B.; Lin, A. Fully 
Planarized Perfect Metamaterial Absorbers With No Photonic Nanostructures. IEEE Photonics J. 2016, 8 (1), 2200109.

(104) Fu, S. M.; Zhong, Y. K.; Tu, M. H.; Chen, B. R.; Lin, A. A Fully Functionalized Metamaterial Perfect Absorber with Simple Design and Implementation. Sci. Rep. 2016, 6, 36244.

(105) Ghobadi, A.; Dereshgi, S. A.; Hajian, H.; Bozok, B.; Butun, B.; Ozbay, E. Ultra-Broadband, Wide Angle Absorber Utilizing Metal Insulator Multilayers Stack with a Multi- Thickness Metal Surface Texture. Sci. Rep. 2017, 7, 4755.

(106) Ghobadi, A.; Hajian, H.; Dereshgi, S. A.; Bozok, B.; Butun, B.; Ozbay, E. Disordered Nanohole Patterns in Metal-Insulator Multilayer for Ultra-Broadband Light Absorption: Atomic Layer Deposition for Lithography Free Highly Repeatable Large Scale Multilayer Growth. Sci. Rep. 2017, 7, 15079.

(107) Abedini Dereshgi, S.; Ghobadi, A.; Hajian, H.; Butun, B.; Ozbay, E. Ultra-Broadband, Lithography-Free, and Large-Scale Compatible Perfect Absorbers: The Optimum Choice of Metal Layers in Metal-Insulator Multilayer Stacks. Sci. Rep. 2017, 7 (1), na.

(108) Park, C. S.; Shrestha, V. R.; Lee, S. S.; Kim, E. S.; Choi, D. Y.

Omnidirectional Color Filters Capitalizing on a Nano-Resonator of Ag-TiO2 -Ag Integrated with a Phase Compensating Dielectric Overlay. Sci. Rep. 2015, 5, 8467.

(109) Lee, K.-T.; Seo, S.; Lee, J. Y.; Guo, L. J. Strong Resonance Effect in a Lossy Medium-Based Optical Cavity for Angle Robust Spectrum Filters. Adv. Mater. 2014, 26 (36), 6324-6328.

(110) Han, J. H.; Kim, D.-Y.; Kim, D.; Choi, K. C. Highly Conductive and Flexible Color Filter Electrode Using Multilayer Film Structure. Sci. Rep. 2016, 6, 29341.

(111) Lee, K. T.; Seo, S.; Yong Lee, J.; Jay Guo, L. Ultrathin MetalSemiconductor-Metal Resonator for Angle Invariant Visible Band Transmission Filters. Appl. Phys. Lett. 2014, 104, 231112.

(112) Yang, C.; Shen, W.; Zhang, Y.; Li, K.; Fang, X.; Zhang, X.; Liu, X. Compact Multilayer Film Structure for Angle Insensitive Color Filtering. Sci. Rep. 2015, 5, 9285.

(113) Lee, K. T.; Han, S. Y.; Park, H. J. Omnidirectional Flexible Transmissive Structural Colors with High-Color-Purity and HighEfficiency Exploiting Multicavity Resonances. Adv. Opt. Mater. 2017, 5 (14), 1700284.

(114) Li, Z.; Butun, S.; Aydin, K. Lithography-Free Transmission Fi Lters at Ultraviolet Frequencies Using Ultra-Thin Aluminum Films. J. Opt. 2016, 18, 065006.

(115) Ghobadi, A.; Hajian, H.; Rashed, A. R.; Butun, B.; Ozbay, E. Tuning the Metal Filling Fraction in Metal-Insulator-Metal UltraBroadband Perfect Absorbers to Maximize the Absorption Bandwidth. Photonics Res. 2018, 6 (3), na.

(116) Palik, E. D. Handbook of Optical Constants of Solids; Academic Press, 1998; Vol. 3.

(117) Toudert, J.; Serna, R.; Camps, I.; Wojcik, J.; Mascher, P.; Rebollar, E.; Ezquerra, T. A. Unveiling the Far Infrared-to-Ultraviolet Optical Properties of Bismuth for Applications in Plasmonics and Nanophotonics. J. Phys. Chem. C 2017, 121 (6), 3511-3521.

(118) Lin, Y.; Chen, W. Perfect Meta-Absorber by Using Pod-like Nanostructures with Ultra-Broadband, Omnidirectional, and Polarization-Independent Characteristics. Sci. Rep. 2018, 8, 7150.

(119) Liu, Z.; Liu, X.; Huang, S.; Pan, P.; Chen, J.; Liu, G.; Gu, G. Automatically Acquired Broadband Plasmonic-Metamaterial Black Absorber during the Metallic Film-Formation. ACS Appl. Mater. Interfaces 2015, 7, 4962-4968.

(120) Yang, C.; Ji, C.; Shen, W.; Lee, K. T.; Zhang, Y.; Liu, X.; Guo, L. J. Compact Multilayer Film Structures for Ultrabroadband, Omnidirectional. ACS Photonics 2016, 3 (4), 590-596.

(121) He, S.; Ding, F.; Mo, L.; Bao, F. Light Absorber with an UltraBroad Flat Band Based on Multi-Sized Slow-Wave Hyperbolic Metamaterial Thin-Films. Prog. Electromagn. Res. 2014, 147, 69-79.

(122) Ji, D.; Song, H.; Zeng, X.; Hu, H.; Liu, K.; Zhang, N.; Gan, Q. Broadband Absorption Engineering of Hyperbolic Metafilm Patterns. Sci. Rep. 2015, 4 (1), 4498.

(123) Eng, H. U. D.; Athai, C. H. J. M.; Angopadhyay, S. H. G.; Ie, J.; Ao, G.; Ang, X. I. Y. Ultra-Broadband Infrared Absorption by
Tapered Hyperbolic Multilayer Waveguides. Opt. Express 2018, 26 (5), 6360-6370.

(124) Cui, Y.; Fung, K. H.; Xu, J.; Ma, H.; Jin, Y.; He, S.; Fang, N. X. Ultrabroadband Light Absorption by a Sawtooth Anisotropic Metamaterial Slab. Nano Lett. 2012, 12 (3), 1443-1447.

(125) Xue, W.; Chen, X.; Peng, Y.; Yang, R. Grating-Type MidInfrared Light Absorber Based on Silicon Carbide Material. Opt. Express 2016, 24 (20), 22596-22605.

(126) Ding, F.; Jin, Y.; Li, B.; Cheng, H.; Mo, L.; He, S. Ultrabroadband Strong Light Absorption Based on Thin Multilayered Metamaterials. Laser Photonics Rev. 2014, 8 (6), 946-953.

(127) Wu, J. Broadband Light Absorption by Tapered MetalDielectric Multilayered Grating Structures. Opt. Commun. 2016, 365, 93-98.

(128) Zhou, J.; Kaplan, A. F.; Chen, L.; Guo, L. J. Experiment and Theory of the Broadband Absorption by a Tapered Hyperbolic Metamaterial Array. ACS Photonics 2014, 1, 618-624.

(129) Ghobadi, A.; Dereshgi, S. A.; Hajian, H.; Birant, G.; Butun, B.; Bek, A.; Ozbay, E. 97\% Light Absorption in an Ultrabroadband Frequency Range Utilizing an Ultrathin Metal Layer: Randomly Oriented, Densely Packed Dielectric Nanowires As an Excellent Light Trapping Scaffold. Nanoscale 2017, 9 (43), 16652-16660.

(130) Lin, A.; Parashar, P.; Yang, C.-C.; Jian, D. R.; Huang, W.-M.; Huang, Y.-W.; Tseng, T.-Y. Lithography-Free Thin-Titanium-Nanocone Metamaterial Perfect Absorbers Using ZnO Nanostructures. Opt. Mater. Express 2017, 7 (10), 3608-3617.

(131) Badugu, R.; Lakowicz, J. R. Tamm State-Coupled Emission: Effect of Probe Location and Emission Wavelength. J. Phys. Chem. C 2014, 118, 21558-21571.

(132) Joannopoulos, J.; Winn, J. N.; Steven, G.; Johnson, R. D. M. Photonic Crystals: Molding the Flow of Light; Princeton University Press, 1995.

(133) Yablonovitch, E. Inhibited Spontaneous Emission in SolidState Physics and Electronics. Phys. Rev. Lett. 1987, 58 (20), 20592062.

(134) Maier, S. A. Plasmonics: Fundamentals and Applications; Springer, 2007.

(135) Yeh, P.; Yariv, A.; Hong, C.; Introduction, I. Electromagnetic Propagation in Periodic Stratified Media. I. General Theory. J. Opt. Soc. Am. 1977, 67 (4), 423-438.

(136) Chen, Y.; Zhang, D.; Qiu, D.; Zhu, L.; Yu, S.; Yao, P.; Wang, P.; Ming, H.; Badugu, R.; Lakowicz, J. R. Back Focal Plane Imaging of Tamm Plasmons and Their Coupled Emission. Laser Photonics Rev. 2014, 8 (6), 933-940.

(137) Chen, Y.; Zhang, D.; Zhu, L.; Fu, Q.; Wang, R.; Wang, P.; Ming, H.; Badugu, R.; Lakowicz, J. R. Effect of Metal Film Thickness on Tamm Plasmon-Coupled Emission. Phys. Chem. Chem. Phys. 2014, $16,25523-25530$.

(138) Choudhury, S. D.; Badugu, R.; Lakowicz, J. R. Directing Fluorescence with Plasmonic and Photonic Structures. Acc. Chem. Res. 2015, 48, 2171-2180.

(139) Kaliteevski, M.; Iorsh, I.; Brand, S.; Abram, R. A.; Chamberlain, J. M.; Kavokin, A. V.; Shelykh, I. A. Tamm PlasmonPolaritons: Possible Electromagnetic States at the Interface of a Metal and a Dielectric Bragg Mirror. Phys. Rev. B: Condens. Matter Mater. Phys. 2007, 76, 165415.

(140) Naik, G. V.; Shalaev, V. M.; Boltasseva, A. Alternative Plasmonic Materials: Beyond Gold and Silver. Adv. Mater. 2013, 25, 3264-3294.

(141) Pizzini, S. Advanced Silicon Materials for Photovoltaic Applications; Wiley, 2012.

(142) Green, M. A.; Hishikawa, Y.; Baillie, A. W. Y. H.; Dunlop, E. D.; Levi, D. H. Solar Cell Efficiency Tables (Version 51). Prog. Photovoltaics 2018, 26, 3-12.

(143) Kats, M. A.; Blanchard, R.; Genevet, P.; Capasso, F. Nanometre Optical Coatings Based on Strong Interference Effects in Highly Absorbing Media. Nat. Mater. 2013, 12 (1), 20-24. 
(144) Rebello, M.; Dias, S.; Gong, C.; Benson, Z. A.; Leite, M. S. Lithography-Free, Omnidirectional, CMOS-Compatible AlCu Alloys for Thin-Film Superabsorbers. Adv. Opt. Mater. 2018, 6, 1700830.

(145) Liu, D.; Yu, H.; Yang, Z.; Duan, Y. Ultrathin Planar Broadband Absorber through Effective Medium Design. Nano Res. 2016, 9 (8), 2354-2363.

(146) Li, Q.; Du, K.; Mao, K.; Fang, X.; Zhao, D.; Ye, H.; Qiu, M. Transmission Enhancement Based on Strong Interference in MetalSemiconductor Layered Film for Energy Harvesting. Sci. Rep. 2016, 6, 29195.

(147) Liu, D.; Yu, H.; Duan, Y.; Li, Q.; Xuan, Y. New Insight into the Angle Insensitivity of Ultrathin Planar Optical Absorbers for Broadband Solar Energy Harvesting. Sci. Rep. 2016, 6, 32515.

(148) Dotan, H.; Kfir, O.; Sharlin, E.; Blank, O.; Gross, M.; Dumchin, I.; Ankonina, G.; Rothschild, A. Resonant Light Trapping in Ultrathin Films for Water Splitting. Nat. Mater. 2013, 12 (2), 158164.

(149) Steenhoff, V.; Theuring, M.; Vehse, M.; von Maydell, K. Ultrathin Resonant-Cavity-Enhanced Solar Cells with Amorphous Germanium Absorbers. Adv. Opt. Mater. 2015, 3, 182-186.

(150) Liu, J.; Xue, Y.; Wang, Z.; Xu, Z.; Zheng, C.; Weber, B.; Song, J.; Wang, Y.; Lu, Y.; Zhang, Y.; Bao, Q. Two-Dimensional CH 3 NH 3 PbI 3 Perovskite: Synthesis and Optoelectronic Application. ACS Nano 2016, 10, 3536-3542.

(151) Zhu, L.; Zhang, H.; Lu, Q.; Wang, Y.; Deng, Z.; Hu, Y.; Lou, Z.; Cui, Q.; Hou, Y.; Teng, F. Synthesis of Ultrathin TwoDimensional Organic - Inorganic Hybrid Perovskite Nanosheets for Polymer Field-Effect Transistors. J. Mater. Chem. C 2018, 6, 3945-3950.

(152) Yang, S.; Niu, W.; Wang, A.; Fan, Z.; Chen, B.; Tan, C.; Lu, Q. Ultrathin Two-Dimensional Organic - Inorganic Hybrid Perovskite Nanosheets with Bright, Tunable Photoluminescence and High Stability. Angew. Chem. 2017, 129, 4316-4319.

(153) Ma, D.; Fu, Y.; Dang, L.; Zhai, J.; Guzei, I. A.; Jin, S. SingleCrystal Microplates of Two-Dimensional Organic - Inorganic Lead Halide Layered Perovskites for Optoelectronics. Nano Res. 2017, 10 (6), 2117-2129.

(154) Zhang, Y.; Liu, W.; Li, Z.; Cheng, H.; Zhang, Y.; Jia, G.; Chen, $S$. Ultrathin Polarization-Insensitive Wide-Angle Broadband nearPerfect Absorber in the Visible Regime Based on Few-Layer MoS 2 Films. Appl. Phys. Lett. 2017, 111, 111109.

(155) Jariwala, D.; Davoyan, A. R.; Tagliabue, G.; Sherrott, M. C.; Wong, J.; Atwater, H. A. Near-Unity Absorption in van Der Waals Semiconductors for Ultrathin Optoelectronics. Nano Lett. 2016, 16, 5482-5487.

(156) Piper, J. R.; Fan, S. Broadband Absorption Enhancement in Solar Cells with an Atomically Thin Active Layer. ACS Photonics 2016, 3, 571-577.

(157) Xia, Z.; Song, H.; Kim, M.; Zhou, M.; Chang, T.; Liu, D.; Yin, X.; Xiong, K.; Mi, H.; Wang, X.; Xia, F.; Yu, Z.; Ma, Z. J.; Gan, Q. Single-Crystalline Germanium Nanomembrane Photodetectors on Foreign Nanocavities. Sci. Adv. 2017, 3, 1-9.

(158) Bouhjar, F.; Bessaïs, B.; Marí, B. Ultrathin-Layer $\alpha-\mathrm{Fe}_{2} \mathrm{O}_{3}$ Deposited under Hematite for Solar Water Splitting. J. Solid State Electrochem. 2018, 22, 2347.

(159) Wang, Y.; Han, J.; Gu, X.; Dimitrijev, S.; Hou, Y.; Zhang, S. Ultrathin Fe2O3 Nanoflakes Using Smart Chemical Stripping for High Performance Lithium Storage. J. Mater. Chem. A 2017, 5 (001), 18737-18743.

(160) Digdaya, I. A.; Trześniewski, B. J.; Adhyaksa, G. W. P.; Garnett, E. C.; Smith, W. A. General Considerations for Improving Photovoltage in Metal - Insulator - Semiconductor Photoanodes. J. Phys. Chem. C 2018, 122, 5462-5471.

(161) Fan, S.; Zheng, X. High-Performance Ultrathin BiVO4 Photoanode on Textured Polydimethylsiloxane Substrates for Solar Water Splitting. ACS Energy Lett. 2016, 1, 68-75.

(162) Ryckman, J. D. Random Coherent Perfect Absorption with 2D Atomic Materials Mediated by Anderson Localization. ACS Photonics 2018, 5, 574-580.
(163) Zheng, J.; Barton, R. A.; Englund, D. Broadband Coherent Absorption in Chirped-Planar-Dielectric Cavities for 2D-MaterialBased Photovoltaics and Photodetectors. ACS Photonics 2014, 1, $768-774$.

(164) Lu, H.; Gan, X.; Mao, D.; Fan, Y.; Yang, D.; Zhao, J. Nearly Perfect Absorption of Light in Monolayer Molybdenum Disulfide Supported by Multilayer Structures. Opt. Express 2017, 25 (18), 21630-21636.

(165) Zhu, C.; Li, J.; Yang, Y.; Huang, J.; Lu, Y.; Zhao, X. SiO ${ }_{2} / \mathrm{Bi}-$ Layer GZO/Ag Structures for near-Infrared Broadband Wide-Angle. J. Phys. D: Appl. Phys. 2016, 49, 425106.

(166) Yoon, J.; Zhou, M.; Badsha, A.; Kim, T. Y.; Jun, Y. C. Broadband Epsilon-Near-Zero Perfect Absorption in the NearInfrared. Sci. Rep. 2015, 5, 12788.

(167) Zheng, H.; Zhang, R.; Li, D.; Chen, X.; Wang, S.; Zheng, Y.; Li, M.; Hu, Z.; Dai, N.; Chen, L. Optical Properties of Al-Doped ZnO Films in the Infrared Region and Their Absorption Applications. Nanoscale Res. Lett. 2018, 13 (149), na.

(168) Zhang, Y.; Wei, T.; Dong, W.; Huang, C.; Zhang, K.; Sun, Y. Near-Perfect Infrared Absorption from Dielectric Multilayer of Plasmonic Aluminum-Doped Zinc Oxide. Appl. Phys. Lett. 2013, $102,213117$.

(169) Liu, J.; Naik, G. V.; Ishii, S.; Devault, C.; Boltasseva, A.; Shalaev, V. M.; Narimanov, E. Optical Absorption of Hyperbolic Metamaterial with Stochastic Surfaces. Opt. Express 2014, 22 (8), 8834-8838.

(170) Novoselov, K. S.; Geim, A. K.; Morozov, S. V.; Jiang, D.; Zhang, Y.; Dubonos, S. V.; Grigorieva, I. V.; Firsov, A. A. Electric Field Effect in Atomically Thin Carbon Films. Science 2004, 306, 666-670.

(171) Grigorenko, A. N.; Polini, M.; Novoselov, K. S. Graphene Plasmonics. Nat. Photonics 2012, 6, 749-758.

(172) Low, T.; Avouris, P. Graphene Plasmonics for Terahertz to Mid-Infrared Applications. ACS Nano 2014, 8 (2), 1086-1101.

(173) Thongrattanasiri, S.; Koppens, F. H. L.; Garc1, F. J. Complete Optical Absorption in Periodically Patterned Graphene. Phys. Rev. Lett. 2012, 108, 047401.

(174) Liu, P. Q.; Valmorra, F.; Maissen, C.; Faist, J. Electrically Tunable Graphene Anti-Dot Array Terahertz Plasmonic Crystals Exhibiting Multi-Band Resonances. Optica 2015, 2 (2), 135-140.

(175) Deng, B.; Guo, Q.; Li, C.; Wang, H.; Ling, X.; Farmer, D. B.; Han, S.; Kong, J.; Xia, F. Coupling-Enhanced Broadband MidInfrared Light Absorption in Graphene Plasmonic Nanostructures. ACS Nano 2016, 10, 11172-11178.

(176) Wang, X.; Jiang, X.; You, Q.; Guo, J.; Dai, X.; Xiang, Y. Tunable and Multichannel Terahertz Perfect Absorber Due to Tamm Surface Plasmons with Graphene. Photonics Res. 2017, 5 (6), 536542.

(177) Low, T.; Rodin, A. S.; Carvalho, A.; Jiang, Y.; Wang, H.; Xia, F.; Neto, A. H. C. Tunable Optical Properties of Multilayer Black Phosphorus Thin Films. Phys. Rev. B: Condens. Matter Mater. Phys. 2014, 90, 075434.

(178) Nemilentsau, A.; Low, T.; Hanson, G. Anisotropic 2D Materials for Tunable Hyperbolic Plasmonics. Phys. Rev. Lett. 2016, 116, 066804.

(179) Ali, S.; Gangaraj, H.; Member, S.; Low, T.; Nemilentsau, A.; Hanson, G. W. Directive Surface Plasmons on Tunable TwoDimensional Hyperbolic Metasurfaces and Black Phosphorus: Green' s Function and Complex Plane Analysis. IEEE Trans. Antennas Propag. 2017, 65 (3), 1174-1186.

(180) Liu, Z.; Aydin, K. Localized Surface Plasmons in Nanostructured Monolayer Black Phosphorus. Nano Lett. 2016, 16, 34573462.

(181) Wang, J.; Jiang, Y. Infrared Absorber Based on Sandwiched Two- Dimensional Black Phosphorus Metamaterials. Opt. Express 2017, 25 (5), 5206-5216.

(182) Wang, J.; Jiang, Y.; Hu, Z. Dual-Band and PolarizationIndependent Infrared Absorber Based on Two-Dimensional Black Phosphorus Metamaterials. Opt. Express 2017, 25 (18), 226-232. 
(183) Song, X.; Liu, Z.; Xiang, Y.; Aydin, K. Biaxial Hyperbolic Metamaterials Using Anisotropic Few-Layer Black Phosphorus. Opt. Express 2018, 26 (5), 971-978.

(184) Zhang, Y.; Small, J. P.; Pontius, W. V.; Kim, P.; Zhang, Y.; Small, J. P.; Pontius, W. V.; Kim, P. Fabrication and Electric-FieldDependent Transport Measurements of Mesoscopic Graphite Devices. Appl. Phys. Lett. 2005, 86, 073104.

(185) Novoselov, K. S.; Geim, A. K.; Morozov, S. V.; Jiang, D.; Katsnelson, M. I.; Grigorieva, I. V.; Dubonos, S. V. Two-Dimensional Gas of Massless Dirac Fermions in Graphene. Nature 2005, 438, 197-200.

(186) Chang, Y.; Liu, C.; Liu, C.; Zhang, S.; Marder, S. R.; Narimanov, E. E.; Zhong, Z.; Norris, T. B. Realization of Mid-Infrared Graphene Hyperbolic Metamaterials. Nat. Commun. 2016, 7, 10568.

(187) Othman, M. A. K.; Guclu, C.; Capolino, F. Graphene-Based Tunable Hyperbolic Metamaterials and Enhanced near-Field Absorption. Opt. Express 2013, 21 (6), 7614-7632.

(188) Ning, R.; Liu, S.; Zhang, H.; Bian, B.; Kong, X. Tunable Absorption in Graphene-Based Hyperbolic Metamaterials for MidInfrared Range. Phys. B 2015, 457, 144-148.

(189) Zhu, J.; Yan, S.; Feng, N.; Ye, L.; Ou, J.; Liu, Q. H. Near Unity Ultraviolet Absorption in Graphene without Patterning. Appl. Phys. Lett. 2018, 112, 153106.

(190) Caldwell, J. D.; Lindsay, L.; Giannini, V.; Vurgaftman, I.; Reinecke, T. L.; Maier, S. A.; Glembocki, O. J. Low-Loss, Infrared and Terahertz Nanophotonics Using Surface Phonon Polaritons. Nanophotonics 2014, 4, 1-26.

(191) Lee, B. J.; Fu, C. J.; Zhang, Z. M. Coherent Thermal Emission from One-Dimensional Photonic Crystals. Appl. Phys. Lett. 2005, 87, 071904.

(192) Shi, K.; Bao, F.; He, S. Enhanced Near-Field Thermal Radiation Based on Multilayer Graphene-HBN Heterostructures. ACS Photonics 2017, 4, 971-978.

(193) Hajian, H.; Ghobadi, A.; Dereshgi, S. A.; Butun, B.; Ozbay, E. Hybrid Plasmon-Phonon Polariton Bands in Graphene-Hexagonal Boron Nitride Metamaterials [Invited]. J. Opt. Soc. Am. B 2017, 34 (7), na.

(194) Dai, S.; Fei, Z.; Ma, Q.; Rodin, A. S.; Wagner, M.; McLeod, A. S.; Liu, M. K.; Gannett, W.; Regan, W.; Watanabe, K.; Taniguchi, T.; Thiemens, M.; Dominguez, G.; Neto, A. H. C.; Zettl, A.; Keilmann, F.; Jarillo-Herrero, P.; Fogler, M. M.; Basov, D. N. Tunable Phonon Polaritons in Atomically Thin van Der Waals Crystals of Boron Nitride. Science (Washington, DC, U. S.) 2014, 343, 1125-1130.

(195) Hajian, H.; Ghobadi, A.; Butun, B.; Ozbay, E. Tunable, Omnidirectional, and Nearly Perfect Resonant Absorptions by a Graphene-HBN- Based Hole Array Metamaterial. Opt. Express 2018, 26 (13), 16940-16954.

(196) Kumar, A.; Low, T.; Fung, K. H.; Avouris, P.; Fang, N. X. Tunable Light - Matter Interaction and the Role of Hyperbolicity in Graphene - HBN System. Nano Lett. 2015, 15, 3172-3180.

(197) Dai, S.; Ma, Q.; Liu, M. K.; Andersen, T.; Fei, Z.; Gold, M. D.; Wagner, M.; Watanabe, K.; Taniguchi, T.; Thiemens, M.; Keilmann, F.; Janssen, G. C. A. M.; Zhu, S. Graphene on Hexagonal Boron Nitride as a Tunable Hyperbolic Metamaterial. Nat. Nanotechnol. 2015, 10, 682-687.

(198) Zhao, B.; Zhang, Z. M. Resonance Perfect Absorption by Exciting Hyperbolic Phonon Polaritons in 1D HBN Gratings. Opt. Express 2017, 25 (7), 7791-7796.

(199) Zhao, B.; Zhang, Z. M. Perfect Mid-Infrared Absorption by Hybrid Phonon-Plasmon Polaritons in HBN/Metal-Grating Anisotropic Structures. Int. J. Heat Mass Transfer 2017, 106, 1025-1034.

(200) Li, P.; Dolado, I.; Casanova, F.; Hueso, L. E.; Hillenbrand, R. Optical Nanoimaging of Hyperbolic Surface Polaritons at the Edges of van Der Waals Materials. Nano Lett. 2017, 17, 228-235.

(201) Alfaro-Mozaz, F. J.; Alonso-Gonzalez, P.; Ve, S.; Dolado, I.; Autore, M.; Mastel, S.; Casanova, F.; Hueso, L. E.; Li, P.; Nikitin, A. Y.; Hillenbrand, R. Nanoimaging of Resonating Hyperbolic Polaritons in Linear Boron Nitride Antennas. Nat. Commun. 2017, 8, 15624.
(202) Yoxall, E.; Schnell, M.; Nikitin, A. Y.; Txoperena, O.; Woessner, A.; Lundeberg, M. B.; Casanova, F.; Hueso, L. E.; Koppens, F. H. L.; Hillenbrand, R. Direct Observation of Ultraslow Hyperbolic Polariton Propagation with Negative Phase Velocity. Nat. Photonics 2015, 9, 674-679.

(203) Li, P.; Dolado, I.; Alfaro-mozaz, F. J.; Casanova, F.; Hueso, L. E.; Liu, S.; Edgar, J. H.; Nikitin, A. Y.; Vélez, S.; Hillenbrand, R. Infrared Hyperbolic Metasurface Based on Nanostructured van Der Waals Materials. Science (Washington, DC, U. S.) 2018, 359, 892-896.

(204) Hillenbrand, R.; Taubner, T.; K, F. Phonon-Enhanced Lightmatter Interaction at the Nanometre Scale. Nature 2002, 418, 159162.

(205) Carminati, Â.; Joulain, K. Coherent Emission of Light by Thermal Sources. Nature 2002, 416, 61-64.

(206) Taubner, T.; Korobkin, D.; Urzhumov, Y.; Shvets, G.; Hillenbrand, R. Near-Field Microscopy Through a SiC Superlens. Science (Washington, DC, U. S.) 2006, 313, 1595.

(207) Schuller, J. A.; Taubner, T.; Brongersma, M. L. Optical Antenna Thermal Emitters. Nat. Photonics 2009, 3 (11), 658-661.

(208) Lee, B. J.; Zhang, Z. M. Coherent Thermal Emission From Modified Periodic Multilayer Structures. J. Heat Transfer 2007, 129, $17-26$.

(209) Shelton, D. J.; Peters, D. W.; Sinclair, M. B.; Brener, I.; Warne, L. K.; Basilio, L. I. Effect of Thin Silicon Dioxide Layers on Resonant Frequency in Infrared Metamaterials. Opt. Express 2010, 18 (2), $1085-1090$

(210) Ng, S. S.; Hassan, Z.; Hassan, H. A. Experimental and Theoretical Studies of Surface Phonon Polariton of AlN Thin Film. Appl. Phys. Lett. 2007, 90, 081902.

(211) Kafesaki, M.; Basharin, A. A.; Economou, E. N.; Soukoulis, C. M. THz Metamaterials Made of Phonon-Polariton Materials. Photonics Nanostructures - Fundam. Appl. 2014, 12, 376-386.

(212) Serebryannikov, A. E.; Ozbay, E.; Nojima, S. Asymmetric Transmission of Terahertz Waves Using Polar Dielectrics. Opt. Express 2014, 22 (3), 3075-3088.

(213) Feng, K.; Streyer, W.; Islam, S. M.; Verma, J.; Jena, D.; Wasserman, D.; Anthony, J.; Feng, K.; Streyer, W.; Islam, S. M.; Verma, J.; Jena, D. Localized Surface Phonon Polariton Resonances in Polar Gallium Nitride. Appl. Phys. Lett. 2015, 107, 081108.

(214) Serebryannikov, A. E.; Nojima, S.; Alici, K. B.; Ozbay, E. Photonic Crystals Made of Polar Dielectrics Effect of In-Material Losses on Terahertz Absorption, Transmission, and Reflection in Photonic Crystals Made of Polar Dielectrics. J. Appl. Phys. 2015, 118, 133101.

(215) Jung, J. Y.; Song, K.; Choi, J. H.; Lee, J.; Choi, D. G.; Jeong, J. H.; Neikirk, D. P. Infrared Broadband Metasurface Absorber for Reducing the Thermal Mass of a Microbolometer. Sci. Rep. 2017, 7 (1), 1-8.

(216) Gou, J.; Wang, J.; Li, W.; Tai, H.; Gu, D.; Jiang, Y. Terahertz Absorption Characteristics of NiCr Film and Enhanced Absorption by Reactive Ion Etching in a Microbolometer Focal Plane Array. J. Infrared, Millimeter, Terahertz Waves 2013, 34 (7-8), 431-436.

(217) Hajian, H.; Ghobadi, A.; Dereshgi, S. A.; Butun, B.; Ozbay, E. Hybrid Plasmon - Phonon Polariton Bands in Graphene Hexagonal Boron Nitride Metamaterials [ Invited ]. J. Opt. Soc. Am. B 2017, 34 (7), D29-D35.

(218) Wu, J.; Jiang, L.; Guo, J.; Dai, X.; Xiang, Y.; Wen, S. Tunable Perfect Absorption at Infrared Frequencies by a Graphene-HBN Hyper Crystal. Opt. Express 2016, 24 (15), 17103-17114.

(219) Rensberg, J.; Zhou, Y.; Richter, S.; Wan, C.; Zhang, S.; Schöppe, P.; Schmidt-grund, R.; Ramanathan, S.; Capasso, F.; Kats, M. A.; Ronning, C. Epsilon-Near-Zero Substrate Engineering for Ultrathin-Film Perfect Absorbers. Phys. Rev. Appl. 2017, 8, 014009.

(220) Farhat, M.; Cheng, T.-C.; Le, K. Q.; Cheng, M. M.-C.; Bağc1, H.; Chen, P.-Y. Mirror-Backed Dark Alumina: A Nearly Perfect Absorber for Thermoelectronics and Thermophotovotaics. Sci. Rep. 2016, 6, 19984. 
(221) Jariwala, D.; Davoyan, A. R.; Wong, J.; Atwater, H. A. Van Der Waals Materials for Atomically-Thin Photovoltaics: Promise and Outlook. ACS Photonics 2017, 4, 2962-2970.

(222) Li, P.; Lewin, M.; Kretinin, A. V.; Caldwell, J. D.; Novoselov, K. S.; Taniguchi, T.; Watanabe, K.; Gaussmann, F.; Taubner, T. Hyperbolic Phonon-Polaritons in Boron Nitride for near-Field Optical Imaging and Focusing. Nat. Commun. 2015, 6, 7507.

(223) Yu, Z.; Veronis, G.; Fan, S.; Brongersma, M. L.; Yu, Z.; Veronis, G.; Fan, S. Design of Midinfrared Photodetectors Enhanced by Surface Plasmons on Grating Structures Design of Midinfrared Photodetectors Enhanced by Surface Plasmons. Appl. Phys. Lett. 2006, 89,151116

(224) Lochbaum, A.; Fedoryshyn, Y.; Dorodnyy, A.; Koch, U.; Hafner, C. On-Chip Narrowband Thermal Emitter for Mid-IR Optical Gas Sensing. ACS Photonics 2017, 4, 1371-1380. 